\title{
TIME-FREQUENCY ANALYSIS ASSOCIATED WITH THE LAGUERRE WAVELET TRANSFORM
}

\author{
HATEM MEJJAOLI AND KHALIFA TRIMÈCHE
}

Abstract. We define the localization operators associated with Laguerre wavelet transforms. Next, we prove the boundedness and compactness of these operators, which depend on a symbol and two admissible wavelets on $L_{\alpha}^{p}(\mathbb{K}), 1 \leq p \leq \infty$.

\section{INTRODUCTION} law

Let $\mathbb{H}_{d}$ be the $(2 d+1)$-dimensional Heisenberg group with the multiplication

$$
(z, t)\left(z^{\prime}, t^{\prime}\right)=\left(z+z^{\prime}, t+t^{\prime}-\operatorname{Im}\left(z z^{\prime}\right)\right) .
$$

Then $T=\frac{\partial}{\partial t}$ and

$$
Z_{j}=\frac{\partial}{\partial z_{j}}-i \bar{z}_{j} \frac{\partial}{\partial t}, \quad \overline{Z_{j}}=\frac{\partial}{\partial \bar{z}_{j}}+i \overline{z_{j}} \frac{\partial}{\partial t}, \quad j=1, \ldots, d
$$

forms a basis of the left invariant vector fields of $h_{d}^{c}$, the complexification of the Lie algebra $h_{d}$ of $\mathbb{H}_{d}$, where

$$
\frac{\partial}{\partial z_{j}}=\frac{\partial}{\partial x_{j}}-i \frac{\partial}{\partial y_{j}}, \quad \frac{\partial}{\partial \bar{z}_{j}}=\frac{\partial}{\partial x_{j}}+i \frac{\partial}{\partial y_{j}} .
$$

Set

$$
X_{j}=\frac{\partial}{\partial x_{j}}-i y_{j} \frac{\partial}{\partial t}, \quad Y_{j}=\frac{\partial}{\partial y_{j}}+i x_{j} \frac{\partial}{\partial t}, \quad j=1, \ldots, d .
$$

Thus $X_{1}, \ldots, X_{d}, Y_{1}, \ldots, Y_{d}, T$ is a basis of $h_{d}$. A function $f$ on $\mathbb{H}_{d}$ is said to be radial if it is invariant under the action of the unitary group $U(d)$. Let

$$
L_{\text {rad }}^{p}\left(\mathbb{H}_{d}\right):=\left\{f \in L^{p}\left(\mathbb{H}_{d}\right): f(u z, t)=f(z, t) \text { for all } u \in U(d)\right\} .
$$

The theory of harmonic analysis on $L_{\text {rad }}^{p}\left(\mathbb{H}_{d}\right)$ was exploited by many authors (see [23, 27, 32]). When one considers the problems of radial functions on the Heisenberg group $\mathbb{H}_{d}$, the underlying manifold can be regarded as the Laguerre hypergroup $\mathbb{K}:=[0, \infty) \times \mathbb{R}$. Stempak [33] introduced a generalized translation operator on $\mathbb{K}$

2020 Mathematics Subject Classification. 33E30, 42B10, 43A32, 44A20.

Key words and phrases. Laguerre hypergroup; generalized Fourier transform; Laguerre wavelet transform; localization operators; admissible wavelets. 
and established the theory of harmonic analysis on $L^{2}\left(\mathbb{K}, d \nu_{\alpha}\right)$, where the weighted Lebesgue measure $\nu_{\alpha}$ on $\mathbb{K}$ is given by

$$
d \nu_{\alpha}(x, t):=\frac{x^{2 \alpha+1} d x d t}{\pi \Gamma(\alpha+1)}, \quad \alpha \geq 0 .
$$

In this paper we are interested in the Laguerre hypergroup $\mathbb{K}$. We recall that $\left(\mathbb{K}, *_{\alpha}\right)$ is a commutative hypergroup [29, on which the involution and the Haar measure are respectively given by the homeomorphism $(x, t) \rightarrow(x, t)^{-}=(x,-t)$ and the Radon positive measure $d \nu_{\alpha}(x, t)$. The unit element of $\left(\mathbb{K}, *_{\alpha}\right)$ is given by $e=(0,0)$.

The dual of a hypergroup is the space of all bounded continuous and multiplicative functions $\chi$ such that $\bar{\chi}=\chi$. The dual of the Laguerre hypergroup $\hat{\mathbb{K}}$ can be topologically identified with the so-called Heisenberg fan 14, i.e., the subset embedded in $\mathbb{R}^{2}$ given by

$$
\bigcup_{j \in \mathbb{N}}\left\{(\lambda, \mu) \in \mathbb{R}^{2}: \mu=|\lambda|(2 j+\alpha+1), \lambda \neq 0\right\} \cup\left\{(0, \mu) \in \mathbb{R}^{2}: \mu \geq 0\right\} .
$$

Moreover, the subset $\left\{(0, \mu) \in \mathbb{R}^{2}: \mu \geq 0\right\}$ has zero Plancherel measure, and therefore it will be usually disregarded. Following [29], in this paper we identify the dual of the Laguerre hypergroup by $\hat{\mathbb{K}}:=\mathbb{R} \times \mathbb{N}$.

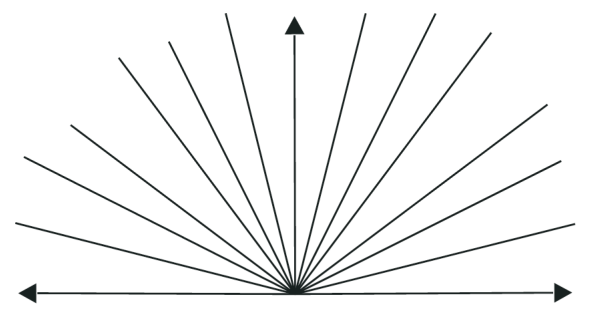

Figure 1. Heisenberg fan

Very recently, the Fourier analysis on $\mathbb{K}$ has been extensively studied with respect to several problems already studied for the Fourier transform; for instance, Radon transform [29], Hardy's inequality [1], functional spaces [2, 19], Littlewood-Paley $g$-functions [20, uncertainty principles [3], Titchmarsh's theorems [28, wavelet multipliers [24, Laguerre-Wigner transform [25], and so on.

In the classical setting, the notion of wavelets was first introduced by Morlet, a French petroleum engineer at Elf Aquitaine, in connection with his study of seismic traces. The mathematical foundations were given by Grossmann and Morlet in [18. The harmonic analyst Meyer and many other mathematicians became aware of this theory and recognized many classical results inside it (see [6, 21, 26]). Classical wavelets have wide applications, ranging from signal analysis in geophysics and acoustics to quantum theory and pure mathematics (see [8, 16] and the references therein). 
Next, the theory of wavelets and the continuous wavelet transform has been extended to hypergroups, in particular to the Laguerre hypergroups (see [29, 34]).

One of the aims of wavelet theory is the study of localization operators for the continuous wavelet transform.

Time-frequency localization operators are a mathematical tool to define a restriction of functions to a region in the time-frequency plane that is compatible with the uncertainty principle and to extract time-frequency features. In this sense, these operators have been introduced and studied by Daubechies [9, 10, 11] and Ramanathan and Topiwala [30, and they are now extensively investigated as an important mathematical tool in signal analysis and other applications [17, 12, 13, 35, 7.

As the harmonic analysis on the Laguerre hypergroup has known remarkable development, it is natural to ask whether there exists the equivalent of the theory of localization operators for the continuous wavelet transform related to this harmonic analysis.

Using the properties of the generalized Fourier transform on the Laguerre hypergroup $\mathbb{K}$, our main aim in this paper is to expose and study the two-wavelet localization operator on the Laguerre hypergroup.

The reason for the extension from one wavelet to two wavelets comes from the extra degree of flexibility in signal analysis and imaging when the localization operators are used as time-varying filters. It turns out that localization operators with two admissible wavelets have a richer mathematical structure than the onewavelet analogues.

The remainder of this paper is arranged as follows. Section 2 contains some basic facts about the Laguerre hypergroup, its dual, and the Schatten-von Neumann classes. In Section 3 we introduce and study the two-wavelet localization operators in the setting of the Laguerre hypergroup. More precisely, the Schatten-von Neumann properties of these two localization wavelet operators are established, and for trace class Laguerre two-wavelet localization operators, the traces and the trace class norm inequalities are presented. Section 4 is devoted to proving that under suitable conditions on the symbols and two admissible wavelets, the $L^{p}$ boundedness and compactness of these two-wavelet localization operators hold.

\section{Preliminaries}

In this section we set some notation and we recall some basic results in harmonic analysis related to Laguerre hypergroups and Schatten-von Neumann classes. The main references are [29, 35].

\subsection{Harmonic analysis on the Laguerre hypergroup.}

\section{Notation:}

- $\mathbb{K}:=[0, \infty) \times \mathbb{R}$ equipped with the weighted Lebesgue measure $\nu_{\alpha}$ on $\mathbb{K}$ given by

$$
d \nu_{\alpha}(x, t):=\frac{x^{2 \alpha+1} d x d t}{\pi \Gamma(\alpha+1)}, \quad \alpha \geq 0 .
$$


- For $p \in[1, \infty], p^{\prime}$ denotes the conjugate exponent of $p$.

- $L_{\alpha}^{p}(\mathbb{K}), 1 \leq p \leq \infty$, is the space of measurable functions on $\mathbb{K}$ satisfying

$$
\begin{aligned}
\|f\|_{L_{\alpha}^{p}(\mathbb{K})} & =\left(\int_{\mathbb{K}}|f(x, t)|^{p} d \nu_{\alpha}(x, t)\right)^{1 / p}<\infty, \quad 1 \leq p<\infty, \\
\|f\|_{L_{\alpha}^{\infty}(\mathbb{K})} & =\operatorname{ess~sup}_{(x, t) \in \mathbb{K}}|f(x, t)|<\infty .
\end{aligned}
$$

- $C_{*}(\mathbb{K})$ is the space of continuous functions on $\mathbb{R}^{2}$, even with respect to the first variable.

- $C_{*, c}(\mathbb{K})$ is the subspace of $C_{*}(\mathbb{K})$ formed by functions with compact support.

- $\mathcal{L}_{m}^{(\alpha)}$ is the Laguerre function defined on $[0, \infty)$ by

$$
\mathcal{L}_{m}^{(\alpha)}(x)=e^{-\frac{x}{2}} L_{m}^{(\alpha)}(x) / L_{m}^{(\alpha)}(0),
$$

$L_{m}^{(\alpha)}$ being the Laguerre polynomial of degree $m$ and order $\alpha$.

- $\hat{\mathbb{K}}:=\mathbb{R} \times \mathbb{N}$ equipped with the weighted Lebesgue measure $\gamma_{\alpha}$ on $\hat{\mathbb{K}}$ given by

$$
\int_{\widehat{\mathbb{K}}} g(\lambda, m) d \gamma_{\alpha}(\lambda, m)=\sum_{m=0}^{\infty} L_{m}^{(\alpha)}(0) \int_{\mathbb{R}} g(\lambda, m)|\lambda|^{\alpha+1} d \lambda .
$$

- $L_{\alpha}^{p}(\hat{\mathbb{K}}), p \in[1, \infty]$, is the space of measurable functions $g: \hat{\mathbb{K}} \rightarrow \mathbb{C}$ such that $\|g\|_{L_{\alpha}^{p}(\hat{\mathbb{K}})}<\infty$, where

$$
\begin{aligned}
\|g\|_{L_{\alpha}^{p}(\hat{\mathbb{K}})} & =\left(\int_{\hat{\mathbb{K}}}|g(\lambda, m)|^{p} d \gamma_{\alpha}(\lambda, m)\right)^{\frac{1}{p}}<\infty, \quad 1 \leq p<\infty, \\
\|g\|_{L_{\alpha}^{\infty}(\hat{\mathbb{K}})} & ={\operatorname{ess} \sup _{(\lambda, m) \in \hat{\mathbb{K}}}|g(\lambda, m)|<\infty .},
\end{aligned}
$$

It is well known (see [29]) that for all $(\lambda, m) \in \hat{\mathbb{K}}$, the system

$$
\left\{\begin{aligned}
D_{1} u(x, t) & =i \lambda u(x, t), \quad(x, t) \in \mathbb{K}, \\
D_{2} u(x, t) & =-4|\lambda|\left(m+\frac{\alpha+1}{2}\right) u(x, t), \quad(x, t) \in \mathbb{K}, \\
u(0,0) & =1, \quad \frac{\partial u}{\partial x}(0, t)=0, \quad \forall t \in \mathbb{R},
\end{aligned}\right.
$$

where $D_{1}$ and $D_{2}$ are singular partial differential operators given by

$$
\left\{\begin{array}{l}
D_{1}=\frac{\partial}{\partial t} \\
D_{2}=\frac{\partial^{2}}{\partial x^{2}}+\frac{2 \alpha+1}{x} \frac{\partial}{\partial x}+x^{2} \frac{\partial^{2}}{\partial t^{2}}, \quad(x, t) \in(0, \infty) \times \mathbb{R}
\end{array}\right.
$$

with $\alpha$ a nonnegative number, admits a unique solution $\varphi_{\lambda, m}$ given by

$$
\varphi_{\lambda, m}(x, t)=e^{i \lambda t} \mathcal{L}_{m}^{(\alpha)}\left(|\lambda| x^{2}\right) .
$$

For $\alpha=d-1$, with $d$ a positive integer, the operator $D_{2}$ is the radial part of the sublaplacian on the Heisenberg group $\mathbb{H}_{d}$. 
The harmonic analysis on the Laguerre hypergroup $\mathbb{K}$ is generated by the singular operator

and the norm

$$
\mathcal{L}_{\alpha}:=\frac{\partial^{2}}{\partial x^{2}}+\frac{2 \alpha+1}{x} \frac{\partial}{\partial x}+x^{2} \frac{\partial^{2}}{\partial t^{2}}
$$

$$
N(x, t)=\left(x^{4}+t^{2}\right)^{\frac{1}{4}}, \quad(x, t) \in \mathbb{K},
$$

while its dual $\hat{\mathbb{K}}$ is generated by the differential difference operator

$$
\Lambda=\Lambda_{1}^{2}-\left(2 \Lambda_{2}+2 \frac{\partial}{\partial \lambda}\right)^{2}
$$

where the operators $\Lambda_{1}, \Lambda_{2}$ are given, for a suitable function $g$ on $\hat{\mathbb{K}}$, by

$$
\begin{aligned}
& \Lambda_{1} g(\lambda, m)=\frac{1}{|\lambda|}\left(m \Delta_{+} \Delta_{-} g(\lambda, m)+(\alpha+1) \Delta_{+} g(\lambda, m)\right) \\
& \Lambda_{2} g(\lambda, m)=-\frac{1}{2 \lambda}\left((\alpha+m+1) \Delta_{+} g(\lambda, m)+m \Delta_{-} g(\lambda, m)\right),
\end{aligned}
$$

and the function

$$
\mathcal{N}(\lambda, m)=|\lambda|\left(m+\frac{\alpha+1}{2}\right), \quad(\lambda, m) \in \hat{\mathbb{K}},
$$

where the difference operators $\Delta_{+}, \Delta_{-}$are given, for a suitable function $g$ on $\hat{\mathbb{K}}$, by

$$
\begin{aligned}
& \triangle_{+} g(\lambda, m)=g(\lambda, m+1)-g(\lambda, m), \\
& \triangle_{-} g(\lambda, m)= \begin{cases}g(\lambda, m)-g(\lambda, m-1), & \text { if } m \geq 1 \\
g(\lambda, 0), & \text { if } m=0\end{cases}
\end{aligned}
$$

These operators satisfy some basic properties which can be found in [29, 2]; namely, one has

$$
\begin{aligned}
\mathcal{L}_{\alpha} \varphi_{\lambda, m}(x, t) & =-\mathcal{N}(\lambda, m) \varphi_{\lambda, m}(x, t), \\
\Lambda \varphi_{\lambda, m}(x, t) & =N^{4}(x, t) \varphi_{\lambda, m}(x, t) .
\end{aligned}
$$

Definition 2.1. Let $f \in C_{*, c}(\mathbb{K})$. For all $(x, t)$ and $(y, s)$ in $\mathbb{K}$, we put $\tau_{(x, t)}^{(\alpha)} f(y, s)= \begin{cases}\frac{1}{2 \pi} \int_{0}^{2 \pi} f\left(\sqrt{x^{2}+y^{2}+2 x y \cos \theta}, s+t+x y \sin \theta\right) d \theta, & \text { if } \alpha=0 ; \\ \frac{\alpha}{\pi} \int_{0}^{2 \pi} \int_{0}^{1} f\left(\langle x, y\rangle_{r, \theta}, s+t+x y r \sin \theta\right) r\left(1-r^{2}\right)^{\alpha-1} d r d \theta, & \text { if } \alpha>0,\end{cases}$

where $\left\langle\langle x, y\rangle_{r, \theta}:=\sqrt{x^{2}+y^{2}+2 x y r \cos \theta}\right.$. The operators $\tau_{(x, t)}^{(\alpha)},(x, t) \in \mathbb{K}$, are called generalized translation operators on $\mathbb{K}$.

Proposition 2.2. For all $(\lambda, m) \in \hat{\mathbb{K}}$, the function $\varphi_{\lambda, m}$ satisfies the product formula

$$
\forall(x, t),(y, s) \in \mathbb{K}, \quad \varphi_{\lambda, m}(x, t) \varphi_{\lambda, m}(y, s)=\tau_{(x, t)}^{(\alpha)} \varphi_{\lambda, m}(y, s) .
$$


Corollary 2.3. For all $(\lambda, m) \in \hat{\mathbb{K}}$, the function $\varphi_{\lambda, m}$ is infinitely differentiable on $\mathbb{R}^{2}$, even with respect to the first variable, and satisfies

$$
\sup _{(x, t) \in \mathbb{K}}\left|\varphi_{\lambda, m}(x, t)\right|=1 .
$$

Proposition 2.4. Let $f$ be in $L_{\alpha}^{p}(\mathbb{K}), p \in[1, \infty]$. Then for all $(x, t) \in \mathbb{K}$, the function $\tau_{(x, t)}^{(\alpha)} f$ belongs to $L_{\alpha}^{p}(\mathbb{K})$ and satisfies

$$
\left\|\tau_{(x, t)}^{(\alpha)} f\right\|_{L_{\alpha}^{p}(\mathbb{K})} \leq\|f\|_{L_{\alpha}^{p}(\mathbb{K})} .
$$

Definition 2.5. The generalized convolution product of $f, g \in L_{\alpha}^{1}(\mathbb{K})$ is defined by

$$
f *_{\alpha} g(x, t)=\int_{\mathbb{K}} \tau_{(x, t)}^{\alpha}(\check{f})(s, y) g(s, y) d \nu_{\alpha}(s, y), \quad \text { for all }(x, t) \in \mathbb{K},
$$

with $\check{f}(s, y)=f(s,-y)$.

Proposition 2.6. Let $1 \leq p, q, r \leq \infty$ be such that $\frac{1}{p}+\frac{1}{q}-\frac{1}{r}=1$. If $f$ is a function in $L_{\alpha}^{p}(\mathbb{K})$ and $g$ an element of $L_{\alpha}^{q}(\mathbb{K})$, then $f *_{\alpha} g$ belongs to $L_{\alpha}^{r}(\mathbb{K})$ and we have

$$
\left\|f *_{\alpha} g\right\|_{L_{\alpha}^{r}(\mathbb{K})} \leq\|f\|_{L_{\alpha}^{p}(\mathbb{K})}\|g\|_{L_{\alpha}^{q}(\mathbb{K})} .
$$

\section{Notation:}

- $\mathcal{S}_{*}(\mathbb{K})$ is the space of functions $f: \mathbb{R}^{2} \rightarrow \mathbb{C}$, even with respect to the first variable, $C^{\infty}$ on $\mathbb{R}^{2}$, and rapidly decreasing together with their derivatives, i.e., for all $k, p, q \in \mathbb{N}$ we have

$$
N_{k, p, q}(f)=\sup _{(x, t) \in \mathbb{K}}\left\{\left(1+x^{2}+t^{2}\right)^{k}\left|\frac{\partial^{p+q}}{\partial x^{p} \partial t^{q}} f(x, t)\right|\right\}<\infty .
$$

Equipped with the topology defined by the semi-norms $N_{k, p, q}, \mathcal{S}_{*}(\mathbb{K})$ is a Fréchet space.

- $\mathcal{S}(\hat{\mathbb{K}})$ is the space of functions $g: \hat{\mathbb{K}} \rightarrow \mathbb{C}$ such that

(i) For all $m, p, q, r, s \in \mathbb{N}$, the function

$$
\lambda \mapsto \lambda^{p}\left(|\lambda|\left(m+\frac{\alpha+1}{2}\right)\right)^{q} \Lambda_{1}^{r}\left(\Lambda_{2}+\frac{\partial}{\partial \lambda}\right)^{s} g(\lambda, m)
$$

is bounded and continuous on $\mathbb{R}, C^{\infty}$ on $\mathbb{R}^{*}$ such that the left and the right derivatives at zero exist.

(ii) For all $k, p, q \in \mathbb{N}$ we have

$$
\nu_{k, p, q}(g)=\sup _{(\lambda, m) \in \mathbb{R}^{*} \times \mathbb{N}}\left\{\left(1+\lambda^{2}\left(1+m^{2}\right)\right)^{k}\left|\Lambda_{1}^{p}\left(\Lambda_{2}+\frac{\partial}{\partial \lambda}\right)^{q} g(\lambda, m)\right|\right\}<\infty .
$$

Equipped with the topology defined by the semi-norms $\nu_{k, p, q}, \mathcal{S}(\hat{\mathbb{K}})$ is a Fréchet space.

Definition 2.7. The generalized Fourier transform $\mathcal{F}_{\alpha}$ is defined on $L_{\alpha}^{1}(\mathbb{K})$ by

$$
\mathcal{F}_{\alpha}(f)(\lambda, m)=\int_{\mathbb{K}} \varphi_{-\lambda, m}(x, t) f(x, t) d \nu_{\alpha}(x, t), \quad \text { for all }(\lambda, m) \in \hat{\mathbb{K}} .
$$


Proposition 2.8. Let $f$ be in $L_{\alpha}^{1}(\mathbb{K})$. Then

(i) For all $m \in \mathbb{N}$, the function $\lambda \mapsto \mathcal{F}_{\alpha}(f)(\lambda, m)$ is continuous on $\mathbb{R}$.

(ii) The function $\mathcal{F}_{\alpha}(f)$ is bounded on $\hat{\mathbb{K}}$ and satisfies

$$
\left\|\mathcal{F}_{\alpha}(f)\right\|_{L_{\alpha}^{\infty}(\hat{\mathbb{K}})} \leq\|f\|_{L_{\alpha}^{1}(\mathbb{K})} .
$$

Theorem 2.9. The generalized Fourier transform $\mathcal{F}_{\alpha}$ is a topological isomorphism from $\mathcal{S}_{*}(\mathbb{K})$ onto $\mathcal{S}(\hat{\mathbb{K}})$.

Theorem 2.10. (i) Plancherel's formula for $\mathcal{F}_{\alpha}$ : For all $f$ in $\mathcal{S}_{*}(\mathbb{K})$ we have

$$
\int_{\hat{\mathbb{K}}}\left|\mathcal{F}_{\alpha}(f)(\lambda, m)\right|^{2} d \gamma_{\alpha}(\lambda, m)=\int_{\mathbb{K}}|f(x, t)|^{2} d \nu_{\alpha}(x, t) .
$$

(ii) The generalized Fourier transform $\mathcal{F}_{\alpha}$ extends to an isometric isomorphism from $L_{\alpha}^{2}(\mathbb{K})$ onto $L_{\alpha}^{2}(\hat{\mathbb{K}})$.

Corollary 2.11. For all $f$ and $g$ in $L_{\alpha}^{2}(\mathbb{K})$ we have the following Parseval formula for the generalized Fourier transform $\mathcal{F}_{\alpha}$ :

$$
\int_{\mathbb{K}} f(x, t) \overline{g(x, t)} d \nu_{\alpha}(x, t)=\int_{\widehat{\mathbb{K}}} \mathcal{F}_{\alpha}(f)(\lambda, m) \overline{\mathcal{F}_{\alpha}(g)(\lambda, m)} d \gamma_{\alpha}(\lambda, m) .
$$

Proposition 2.12. (i) For all $f$ and $g$ in $L_{\alpha}^{1}(\mathbb{K})$, we have

$$
\mathcal{F}_{\alpha}(f * g)=\mathcal{F}_{\alpha}(f) \mathcal{F}_{\alpha}(g) .
$$

(ii) Let $f \in L_{\alpha}^{1}(\mathbb{K})$. Then for all $(x, t) \in \mathbb{K}$ and $(\lambda, m) \in \hat{\mathbb{K}}$, we have

$$
\mathcal{F}_{\alpha}\left(\tau_{(x, t)}^{(\alpha)} f\right)(\lambda, m)=\varphi_{\lambda, m}(x, t) \mathcal{F}_{\alpha}(f)(\lambda, m) .
$$

\subsection{Schatten-von Neumann classes.}

\section{Notation:}

- $l^{p}(\mathbb{N}), 1 \leq p \leq \infty$, is the set of all infinite sequences of real (or complex) numbers $u:=\left(u_{j}\right)_{j \in \mathbb{N}}$ such that

$$
\begin{aligned}
\|u\|_{p} & :=\left(\sum_{j=1}^{\infty}\left|u_{j}\right|^{p}\right)^{\frac{1}{p}}<\infty, \quad \text { if } 1 \leq p<\infty, \\
\|u\|_{\infty} & :=\sup _{j \in \mathbb{N}}\left|u_{j}\right|<\infty
\end{aligned}
$$

For $p=2$, we provide the space $l^{2}(\mathbb{N})$ with the scalar product

$$
\langle u, v\rangle_{2}:=\sum_{j=1}^{\infty} u_{j} \overline{v_{j}}
$$

- $B\left(L_{\alpha}^{p}(\mathbb{K})\right), 1 \leq p \leq \infty$, is the space of bounded operators from $L_{\alpha}^{p}(\mathbb{K})$ into itself.

Definition 2.13. (i) The singular values $\left(s_{n}(A)\right)_{n \in \mathbb{N}}$ of a compact operator $A$ in $B\left(L_{\alpha}^{2}(\mathbb{K})\right)$ are the eigenvalues of the positive self-adjoint operator $|A|=\sqrt{A^{*} A}$. 
(ii) For $1 \leq p<\infty$, the Schatten class $S_{p}$ is the space of all compact operators whose singular values lie in $l^{p}(\mathbb{N})$. The space $S_{p}$ is equipped with the norm

$$
\|A\|_{S_{p}}:=\left(\sum_{n=1}^{\infty}\left(s_{n}(A)\right)^{p}\right)^{\frac{1}{p}} .
$$

Remark 2.14. We note that the space $S_{2}$ is the space of Hilbert-Schmidt operators, and $S_{1}$ is the space of trace class operators.

Definition 2.15. The trace of an operator $A$ in $S_{1}$ is defined by

$$
\operatorname{tr}(A)=\sum_{n=1}^{\infty}\left\langle A v_{n}, v_{n}\right\rangle_{L_{\alpha}^{2}(\mathbb{K})}
$$

where $\left(v_{n}\right)_{n}$ is any orthonormal basis of $L_{\alpha}^{2}(\mathbb{K})$.

Remark 2.16. If $A$ is positive, then

$$
\operatorname{tr}(A)=\|A\|_{S_{1}} .
$$

Moreover, a compact operator $A$ on the Hilbert space $L_{\alpha}^{2}(\mathbb{K})$ is Hilbert-Schmidt if the positive operator $A^{*} A$ is in the space of trace class $S_{1}$. Then

$$
\|A\|_{H S}^{2}:=\|A\|_{S_{2}}^{2}=\left\|A^{*} A\right\|_{S_{1}}=\operatorname{tr}\left(A^{*} A\right)=\sum_{n=1}^{\infty}\left\|A v_{n}\right\|_{L_{\alpha}^{2}(\mathbb{K})}^{2}
$$

for any orthonormal basis $\left(v_{n}\right)_{n}$ of $L_{\alpha}^{2}(\mathbb{K})$.

Definition 2.17. We define $S_{\infty}:=B\left(L_{\alpha}^{2}(\mathbb{K})\right)$, equipped with the norm

$$
\|A\|_{S_{\infty}}:=\sup _{\substack{v \in L_{\alpha}^{2}(\mathbb{K}),\|v\|_{L_{\alpha}^{2}(\mathbb{K})}=1}}\|A v\|_{L_{\alpha}^{2}(\mathbb{K})}
$$

2.3. Basic Laguerre wavelet theory. In this subsection we recall some results introduced in [29].

Notation: We denote by $L_{\mu_{\alpha}}^{p}(\mathbb{R} \times \mathbb{K}), p \in[1, \infty]$, the space of measurable functions $f$ on $\mathbb{R} \times \mathbb{K}$ such that

$$
\begin{aligned}
& \|f\|_{L_{\mu_{\alpha}}^{p}(\mathbb{R} \times \mathbb{K})}:=\left(\int_{\mathbb{R} \times \mathbb{K}}|f(a, x, t)|^{p} d \mu_{\alpha}(a, x, t)\right)^{\frac{1}{p}}<\infty, \quad 1 \leq p<\infty, \\
& \|f\|_{L_{\mu_{\alpha}}^{\infty}(\mathbb{R} \times \mathbb{K})}:=\operatorname{ess~sup}_{(a, x, t) \in \mathbb{R} \times \mathbb{K}}|f(a, x, t)|<\infty,
\end{aligned}
$$

where the measure $\mu_{\alpha}$ is defined by

$$
\forall(a, x, t) \in \mathbb{R} \times \mathbb{K}, \quad d \mu_{\alpha}(a, x, t)=\frac{d a d \nu_{\alpha}(x, t)}{|a|^{\alpha+3}} .
$$

Definition 2.18. A Laguerre wavelet on $\mathbb{K}$ is a measurable function $h$ on $\mathbb{K}$ satisfying, for almost all $(\lambda, m) \in \hat{\mathbb{K}} \backslash\{(0,0)\}$, the condition

$$
0<C_{h}:=\int_{\mathbb{R}}\left|\mathcal{F}_{\alpha}(h)(a \lambda, m)\right|^{2} \frac{d a}{|a|}<\infty .
$$


Let $a \in \mathbb{R} \backslash\{0\}$ and let $h$ be a measurable function. We consider the function $h_{a}$ defined by

$$
\forall(x, t) \in \mathbb{K}, \quad h_{a}(x, t):=\frac{1}{|a|^{\alpha+2}} h\left(\frac{x}{\sqrt{|a|}}, \frac{t}{a}\right) .
$$

Proposition 2.19. (i) Let $h \in L_{\alpha}^{p}(\mathbb{K}), p \in[1, \infty]$. The function $h_{a}$ belongs to $L_{\alpha}^{p}(\mathbb{K})$ and we have

$$
\left\|h_{a}\right\|_{L_{\alpha}^{p}(\mathbb{K})}=|a|^{(\alpha+2)\left(\frac{1}{p}-1\right)}\|h\|_{L_{\alpha}^{p}(\mathbb{K})} .
$$

(ii) Let $a \in \mathbb{R} \backslash\{0\}$ and let $h$ be in $L_{\alpha}^{1}(\mathbb{K}) \cup L_{\alpha}^{2}(\mathbb{K})$. We have

$$
\mathcal{F}_{\alpha}\left(h_{a}\right)(\lambda, m)=\mathcal{F}_{\alpha}(h)(a \lambda, a m), \quad(\lambda, m) \in \hat{\mathbb{K}} .
$$

Let $a \in \mathbb{R} \backslash\{0\}$ and let $h$ be in $L_{\alpha}^{p}(\mathbb{K}), p \in[1, \infty]$. We consider the family $h_{a, x, t}$, $(x, t) \in \mathbb{K}$, of Laguerre wavelets on $\mathbb{K}$ in $L_{\alpha}^{p}(\mathbb{K})$ defined by

$$
\forall(s, y) \in \mathbb{K}, \quad h_{a, x, t}(s, y):=|a|^{\frac{\alpha}{2}+1} \tau_{(x, t)}^{(\alpha)} h_{a}(s, y),
$$

where $\tau_{(x, t)}^{\alpha},(x, t) \in \mathbb{K}$, are the generalized translation operators given by 2.1.

Remarks 2.20. (i) Let $h$ be in $L_{\alpha}^{2}(\mathbb{K})$. We have

$$
\forall(a, x, t) \in \mathbb{R} \times \mathbb{K}, \quad\left\|h_{a, x, t}\right\|_{L_{\alpha}^{2}(\mathbb{K})} \leq\|h\|_{L_{\alpha}^{2}(\mathbb{K})} .
$$

(ii) Let $h \in L_{\alpha}^{p}(\mathbb{K}), p \in[1, \infty]$. One has

$$
\forall(a, x, t) \in \mathbb{R} \times \mathbb{K}, \quad\left\|h_{a, x, t}\right\|_{L_{\alpha}^{p}(\mathbb{K})} \leq|a|^{(\alpha+2)\left(\frac{1}{p}-\frac{1}{2}\right)}\|h\|_{L_{\alpha}^{p}(\mathbb{K})} .
$$

Definition 2.21. Let $h$ be a Laguerre wavelet on $\mathbb{K}$ in $L_{\alpha}^{2}(\mathbb{K})$. The Laguerre continuous wavelet transform $\Phi_{h}^{\alpha}$ on $\mathbb{K}$ is defined for regular functions $f$ on $\mathbb{K}$ by $\forall(a, x, t) \in \mathbb{R} \times \mathbb{K}, \quad \Phi_{h}^{\alpha}(f)(a, x, t)=\int_{\mathbb{K}} f(s, y) \overline{h_{a, x, t}(s, y)} d \nu_{\alpha}(s, y)=\left\langle f, h_{a, x, t}\right\rangle_{L_{\alpha}^{2}(\mathbb{K})}$.

This transform can also be written in the form

$$
\Phi_{h}^{\alpha}(f)(a, x, t)=|a|^{\frac{\alpha}{2}+1} \breve{f} *_{\alpha} \overline{h_{a}}(x, t),
$$

where $\check{f}(x, t)=f(x,-t)$ and $*_{\alpha}$ is the generalized convolution product given by 2.2 .

Theorem 2.22 (Plancherel's formula for $\Phi_{h}^{\alpha}$ ). Let $h$ be a Laguerre wavelet on $\mathbb{K}$ in $L_{\alpha}^{2}(\mathbb{K})$. For all $f$ in $L_{\alpha}^{2}(\mathbb{K})$ we have

$$
\int_{\mathbb{K}}|f(x, t)|^{2} d \nu_{\alpha}(x, t)=\frac{1}{C_{h}} \int_{\mathbb{R} \times \mathbb{K}}\left|\Phi_{h}^{\alpha}(f)(a, x, t)\right|^{2} d \mu_{\alpha}(a, x, t) .
$$

Corollary 2.23 (Parseval's relation). Let $h$ be a Laguerre wavelet on $\mathbb{K}$ in $L_{\alpha}^{2}(\mathbb{K})$ and $f_{1}, f_{2}$ in $L_{\alpha}^{2}(\mathbb{K})$. Then, we have

$$
\int_{\mathbb{K}} f_{1}(x, t) \overline{f_{2}(x, t)} d \nu_{\alpha}(x, t)=\frac{1}{C_{h}} \int_{\mathbb{R} \times \mathbb{K}} \Phi_{h}^{\alpha}\left(f_{1}\right)(a, x, t) \overline{\Phi_{h}^{\alpha}\left(f_{2}\right)(a, x, t)} d \mu_{\alpha}(a, x, t) .
$$


Remarks 2.24. (i) If $h \in L_{\alpha}^{p}(\mathbb{K})$ and $f \in L_{\alpha}^{p^{\prime}}(\mathbb{K}), p \in[1, \infty]$, we define the Laguerre continuous wavelet transform $\Phi_{h}^{\alpha}(f)$ by relation (2.8).

(ii) Let $h$ be a Laguerre wavelet in $L_{\alpha}^{2}(\mathbb{K})$. Then from (2.8), Cauchy-Schwarz inequality, and $(2.5)$, for all $f$ in $L_{\alpha}^{2}(\mathbb{K})$ we have

$$
\left\|\Phi_{h}^{\alpha}(f)\right\|_{L_{\mu_{\alpha}}^{\infty}(\mathbb{R} \times \mathbb{K})} \leq\|f\|_{L_{\alpha}^{2}(\mathbb{K})}\|h\|_{L_{\alpha}^{2}(\mathbb{K})} .
$$

(iii) Let $h \in L_{\alpha}^{p}(\mathbb{K}), p \in[1, \infty]$. Then from relations (2.8) and 2.7) and Hölder's inequality, for all $f$ in $L_{\alpha}^{p^{\prime}}(\mathbb{K})$ we have

$$
\forall(a, x, t) \in \mathbb{R} \times \mathbb{K}, \quad\left|\Phi_{h}^{\alpha}(f)(a, x, t)\right| \leq|a|^{(\alpha+2)\left(\frac{1}{2}-\frac{1}{p}\right)}\|f\|_{L_{\alpha}^{p^{\prime}(\mathbb{K})}}\|h\|_{L_{\alpha}^{p}(\mathbb{K})} .
$$

\section{LAGUERRE TWO-WAVELET LOCALIZATION OPERATORS}

In this section we will derive a host of sufficient conditions for the boundedness and Schatten class of the Laguerre two-wavelet localization operators in terms of properties of the symbol $\sigma$ and the windows $h$ and $k$.

\subsection{Preliminaries.}

Definition 3.1. Let $h, k$ be measurable functions on $\mathbb{K}$, and $\sigma$ a measurable function on $\mathbb{R} \times \mathbb{K}$. We define $\mathcal{L}_{h, k}(\sigma)$, the Laguerre two-wavelet localization operator on $L_{\alpha}^{p}(\mathbb{K}), 1 \leq p \leq \infty$, by

$\mathcal{L}_{h, k}(\sigma)(f)(s, y):=\int_{\mathbb{R} \times \mathbb{K}} \sigma(a, x, t) \Phi_{h}^{\alpha}(f)(a, x, t) k_{a, x, t}(s, y) d \mu_{\alpha}(a, x, t), \quad(s, y) \in \mathbb{K}$.

According to the different choices of the symbols $\sigma$ and the different continuities required, we need to impose different conditions on $h$ and $k$, and then we obtain an operator on $L_{\alpha}^{p}(\mathbb{K})$.

It is often more convenient to interpret the definition of $\mathcal{L}_{h, k}(\sigma)$ in a weak sense, that is, for $f$ in $L_{\alpha}^{p}(\mathbb{K}), p \in[1, \infty]$, and $g$ in $L_{\alpha}^{p^{\prime}}(\mathbb{K})$,

$$
\left\langle\mathcal{L}_{h, k}(\sigma)(f), g\right\rangle_{L_{\alpha}^{2}(\mathbb{K})}=\int_{\mathbb{R} \times \mathbb{K}} \sigma(a, x, t) \Phi_{h}^{\alpha}(f)(a, x, t) \overline{\Phi_{k}^{\alpha}(g)(a, x, t)} d \mu_{\alpha}(a, x, t) .
$$

In what follows, such operator $\mathcal{L}_{h, k}(\sigma)$ will be named localization operator, for the sake of simplicity.

Proposition 3.2. Let $p \in[1, \infty)$. Formally, we assume that we have

$$
\mathcal{L}_{h, k}(\sigma): L_{\alpha}^{p}(\mathbb{K}) \rightarrow L_{\alpha}^{p}(\mathbb{K}) .
$$

Then its adjoint is the linear operator $\mathcal{L}_{k, h}(\bar{\sigma}): L_{\alpha}^{p^{\prime}}(\mathbb{K}) \rightarrow L_{\alpha}^{p^{\prime}}(\mathbb{K})$. 
Proof. For all $f$ in $L_{\alpha}^{p}(\mathbb{K})$ and $g$ in $L_{\alpha}^{p^{\prime}}(\mathbb{K})$ it immediately follows from 3.2 that

$$
\begin{aligned}
& \left\langle\mathcal{L}_{h, k}(\sigma)(f), g\right\rangle_{L_{\alpha}^{2}(\mathbb{K})}=\int_{\mathbb{R} \times \mathbb{K}} \sigma(a, x, t) \Phi_{h}^{\alpha}(f)(a, x, t) \overline{\Phi_{k}^{\alpha}(g)(a, x, t)} d \mu_{\alpha}(a, x, t) \\
& =\overline{\int_{\mathbb{R} \times \mathbb{K}} \overline{\sigma(a, x, t)} \Phi_{k}^{\alpha}(g)(a, x, t) \overline{\Phi_{h}^{\alpha}(f)(a, x, t)} d \mu_{\alpha}(a, x, t)} \\
& =\overline{\left\langle\mathcal{L}_{k, h}(\bar{\sigma})(g), f\right\rangle_{L_{\alpha}^{2}(\mathbb{K})}}=\left\langle f, \mathcal{L}_{k, h}(\bar{\sigma})(g)\right\rangle_{L_{\alpha}^{2}(\mathbb{K})} .
\end{aligned}
$$

Thus we get

$$
\mathcal{L}_{h, k}^{*}(\sigma)=\mathcal{L}_{k, h}(\bar{\sigma})
$$

In the rest of this section, $h$ and $k$ will be two Laguerre wavelets on $\mathbb{K}$ such that

$$
\|h\|_{L_{\alpha}^{2}(\mathbb{K})}=\|k\|_{L_{\alpha}^{2}(\mathbb{K})}=1 .
$$

3.2. Boundedness for $\mathcal{L}_{h, k}(\sigma)$ on $S_{\infty}$. The main result of this subsection is the proof that the linear operators

$$
\mathcal{L}_{h, k}(\sigma): L_{\alpha}^{2}(\mathbb{K}) \rightarrow L_{\alpha}^{2}(\mathbb{K})
$$

are bounded for all symbols $\sigma \in L_{\mu_{\alpha}}^{p}(\mathbb{R} \times \mathbb{K}), 1 \leq p \leq \infty$. We first consider this problem for $\sigma$ in $L_{\mu_{\alpha}}^{1}(\mathbb{R} \times \mathbb{K})$ and next in $L_{\mu_{\alpha}}^{\infty}(\mathbb{R} \times \mathbb{K})$, and we then conclude by using interpolation theory.

Proposition 3.3. Let $\sigma$ be in $L_{\mu_{\alpha}}^{1}(\mathbb{R} \times \mathbb{K})$. Then the localization operator $\mathcal{L}_{h, k}(\sigma)$ is in $S_{\infty}$ and we have

$$
\left\|\mathcal{L}_{h, k}(\sigma)\right\|_{S_{\infty}} \leq\|\sigma\|_{L_{\mu_{\alpha}}^{1}(\mathbb{R} \times \mathbb{K})}
$$

Proof. For all functions $f$ and $g$ in $L_{\alpha}^{2}(\mathbb{K})$, we have from relations 3.2 and 2.10 ,

$$
\begin{aligned}
\left|\left\langle\mathcal{L}_{h, k}(\sigma)(f), g\right\rangle_{L_{\alpha}^{2}(\mathbb{K})}\right| & \leq \int_{\mathbb{R} \times \mathbb{K}}|\sigma(a, x, t)|\left|\Phi_{h}^{\alpha}(f)(a, x, t)\right| \overline{\mid \Phi_{k}^{\alpha}(g)(a, x, t)} \mid d \mu_{\alpha}(a, x, t) \\
& \leq\left\|\Phi_{h}^{\alpha}(f)\right\|_{L_{\mu_{\alpha}}^{\infty}(\mathbb{R} \times \mathbb{K})}\left\|\Phi_{k}^{\alpha}(g)\right\|_{L_{\mu_{\alpha}}^{\infty}(\mathbb{R} \times \mathbb{K})}\|\sigma\|_{L_{\mu_{\alpha}}^{1}(\mathbb{R} \times \mathbb{K})} \\
& \leq\|f\|_{L_{\alpha}^{2}(\mathbb{K})}\|g\|_{L_{\alpha}^{2}(\mathbb{K})}\|\sigma\|_{L_{\mu_{\alpha}}^{1}(\mathbb{R} \times \mathbb{K})} .
\end{aligned}
$$

Thus,

$$
\left\|\mathcal{L}_{h, k}(\sigma)\right\|_{S_{\infty}} \leq\|\sigma\|_{L_{\mu_{\alpha}}^{1}(\mathbb{R} \times \mathbb{K})} .
$$

Proposition 3.4. Let $\sigma$ be in $L_{\mu_{\alpha}}^{\infty}(\mathbb{R} \times \mathbb{K})$. Then the localization operator $\mathcal{L}_{h, k}(\sigma)$ is in $S_{\infty}$ and we have

$$
\left\|\mathcal{L}_{h, k}(\sigma)\right\|_{S_{\infty}} \leq \sqrt{C_{h} C_{k}}\|\sigma\|_{L_{\mu_{\alpha}}^{\infty}(\mathbb{R} \times \mathbb{K})}
$$

Proof. For all functions $f$ and $g$ in $L_{\alpha}^{2}(\mathbb{K})$, we have from Hölder's inequality

$$
\begin{aligned}
\left|\left\langle\mathcal{L}_{h, k}(\sigma)(f), g\right\rangle_{L_{\alpha}^{2}(\mathbb{K})}\right| & \leq \int_{\mathbb{R} \times \mathbb{K}}|\sigma(a, x, t)|\left|\Phi_{h}^{\alpha}(f)(a, x, t)\right|\left|\overline{\Phi_{k}^{\alpha}(g)(a, x, t)}\right| d \mu_{\alpha}(a, x, t) \\
& \leq\|\sigma\|_{L_{\mu_{\alpha}}^{\infty}(\mathbb{R} \times \mathbb{K})}\left\|\Phi_{h}^{\alpha}(f)\right\|_{L_{\mu_{\alpha}}^{2}(\mathbb{R} \times \mathbb{K})}\left\|\Phi_{k}^{\alpha}(g)\right\|_{L_{\mu_{\alpha}}^{2}(\mathbb{R} \times \mathbb{K})} .
\end{aligned}
$$

Using Plancherel's formula for $\Phi_{h}^{\alpha}$ and $\Phi_{k}^{\alpha}$, given by relation (2.9), we get

$$
\left|\left\langle\mathcal{L}_{h, k}(\sigma)(f), g\right\rangle_{L_{\alpha}^{2}(\mathbb{K})}\right| \leq \sqrt{C_{h} C_{k}}\|f\|_{L_{\alpha}^{2}(\mathbb{K})}\|g\|_{L_{\alpha}^{2}(\mathbb{K})}\|\sigma\|_{L_{\mu_{\alpha}}^{\infty}(\mathbb{R} \times \mathbb{K})} .
$$


Thus,

$$
\left\|\mathcal{L}_{h, k}(\sigma)\right\|_{S_{\infty}} \leq \sqrt{C_{h} C_{k}}\|\sigma\|_{L_{\mu_{\alpha}}^{\infty}(\mathbb{R} \times \mathbb{K})}
$$

We can now associate a localization operator $\mathcal{L}_{h, k}(\sigma): L_{\alpha}^{2}(\mathbb{K}) \rightarrow L_{\alpha}^{2}(\mathbb{K})$ to every symbol $\sigma$ in $L_{\mu_{\alpha}}^{p}(\mathbb{R} \times \mathbb{K}), 1 \leq p \leq \infty$, and prove that $\mathcal{L}_{h, k}(\sigma)$ is in $S_{\infty}$. The precise result is the following theorem.

Theorem 3.5. Let $\sigma$ be in $L_{\mu_{\alpha}}^{p}(\mathbb{R} \times \mathbb{K}), 1 \leq p \leq \infty$. Then there exists a unique bounded linear operator $\mathcal{L}_{h, k}(\sigma): L_{\alpha}^{2}(\mathbb{K}) \rightarrow L_{\alpha}^{2}(\mathbb{K})$ such that

$$
\left\|\mathcal{L}_{h, k}(\sigma)\right\|_{S_{\infty}} \leq\left(C_{h} C_{k}\right)^{\frac{p-1}{2 p}}\|\sigma\|_{L_{\mu_{\alpha}}^{p}(\mathbb{R} \times \mathbb{K})} .
$$

Proof. Let $f$ be in $L_{\alpha}^{2}(\mathbb{K})$. We consider the operator

$$
\mathcal{T}: L_{\mu_{\alpha}}^{1}(\mathbb{R} \times \mathbb{K}) \cap L_{\mu_{\alpha}}^{\infty}(\mathbb{R} \times \mathbb{K}) \rightarrow L_{\alpha}^{2}(\mathbb{K})
$$

given by

$$
\mathcal{T}(\sigma):=\mathcal{L}_{h, k}(\sigma)(f) .
$$

Then, by Proposition 3.3 and Proposition 3.4

$$
\|\mathcal{T}(\sigma)\|_{L_{\alpha}^{2}(\mathbb{K})} \leq\|f\|_{L_{\alpha}^{2}(\mathbb{K})}\|\sigma\|_{L_{\mu_{\alpha}}^{1}(\mathbb{R} \times \mathbb{K})}
$$

and

$$
\|\mathcal{T}(\sigma)\|_{L_{\alpha}^{2}(\mathbb{K})} \leq \sqrt{C_{h} C_{k}}\|f\|_{L_{\alpha}^{2}(\mathbb{K})}\|\sigma\|_{L_{\mu_{\alpha}}^{\infty}(\mathbb{R} \times \mathbb{K})} .
$$

Therefore, by 3.5, (3.6), and the Riesz-Thorin interpolation theorem (see 31, Theorem 2] and [35, Theorem 2.11]), $\mathcal{T}$ may be uniquely extended to a linear operator on $L_{\mu_{\alpha}}^{p}(\mathbb{R} \times \mathbb{K}), 1 \leq p \leq \infty$, and we have

$$
\left\|\mathcal{L}_{h, k}(\sigma)(f)\right\|_{L_{\alpha}^{2}(\mathbb{K})}=\|\mathcal{T}(\sigma)\|_{L_{\alpha}^{2}(\mathbb{K})} \leq\left(C_{h} C_{k}\right)^{\frac{p-1}{2 p}}\|f\|_{L_{\alpha}^{2}(\mathbb{K})}\|\sigma\|_{L_{\mu_{\alpha}}^{p}(\mathbb{R} \times \mathbb{K})} .
$$

Since (3.7) is true for arbitrary functions $f$ in $L_{\alpha}^{2}(\mathbb{K})$, we obtain the desired result.

3.3. Schatten-von Neumann properties for $\mathcal{L}_{h, k}(\sigma)$. The main result of this subsection is the proof that the localization operator

$$
\mathcal{L}_{h, k}(\sigma): L_{\alpha}^{2}(\mathbb{K}) \rightarrow L_{\alpha}^{2}(\mathbb{K})
$$

is in the Schatten class $S_{p}$.

Proposition 3.6. Let $\sigma$ be in $L_{\mu_{\alpha}}^{1}(\mathbb{R} \times \mathbb{K})$. Then the localization operator $\mathcal{L}_{h, k}(\sigma)$ is in $S_{2}$ and we have

$$
\left\|\mathcal{L}_{h, k}(\sigma)\right\|_{S_{2}} \leq\|\sigma\|_{L_{\mu_{\alpha}}^{1}(\mathbb{R} \times \mathbb{K})}
$$

Proof. Let $\left\{\phi_{j}, j=1,2, \ldots\right\}$ be an orthonormal basis for $L_{\alpha}^{2}(\mathbb{K})$. Then by 3.2 , Fubini's theorem, Parseval's identity, and the relations 2.8 and (3.3), we have

$$
\begin{aligned}
\sum_{j=1}^{\infty}\left\|\mathcal{L}_{h, k}(\sigma)\left(\phi_{j}\right)\right\|_{L_{\alpha}^{2}(\mathbb{K})}^{2} & =\sum_{j=1}^{\infty}\left\langle\mathcal{L}_{h, k}(\sigma)\left(\phi_{j}\right), \mathcal{L}_{h, k}(\sigma)\left(\phi_{j}\right)\right\rangle_{L_{\alpha}^{2}(\mathbb{K})} \\
& =\int_{\mathbb{R} \times \mathbb{K}} \sigma(a, x, t)\left\langle\mathcal{L}_{h, k}^{*}(\sigma) k_{a, x, t}, h_{a, x, t}\right\rangle_{L_{\alpha}^{2}(\mathbb{K})} d \mu_{\alpha}(a, x, t) .
\end{aligned}
$$


Thus, from $3.3,3.4$, and 2.6 we get

$$
\begin{aligned}
\sum_{j=1}^{\infty}\left\|\mathcal{L}_{h, k}(\sigma)\left(\phi_{j}\right)\right\|_{L_{\alpha}^{2}(\mathbb{K})}^{2} & \leq \int_{\mathbb{R} \times \mathbb{K}}|\sigma(a, x, t)|\left\|\mathcal{L}_{h, k}^{*}(\sigma)\right\|_{S_{\infty}} d \mu_{\alpha}(a, x, t) \\
& \leq\|\sigma\|_{L_{\mu_{\alpha}}^{1}(\mathbb{R} \times \mathbb{K})}^{2}<\infty .
\end{aligned}
$$

So, by $(3.8)$ and Wong's result [35, Prop. 2.8], $\mathcal{L}_{h, k}(\sigma): L_{\alpha}^{2}(\mathbb{K}) \rightarrow L_{\alpha}^{2}(\mathbb{K})$ is in the Hilbert-Schmidt class $S_{2}$ and hence compact.

Proposition 3.7. Let $\sigma$ be a symbol in $L_{\mu_{\alpha}}^{p}(\mathbb{R} \times \mathbb{K}), 1 \leq p<\infty$. Then the localization operator $\mathcal{L}_{h, k}(\sigma)$ is compact.

Proof. Let $\sigma$ be in $L_{\mu_{\alpha}}^{p}(\mathbb{R} \times \mathbb{K})$ and let $\left(\sigma_{n}\right)_{n \in \mathbb{N}}$ be a sequence of functions in

$$
L_{\mu_{\alpha}}^{1}(\mathbb{R} \times \mathbb{K}) \cap L_{\mu_{\alpha}}^{\infty}(\mathbb{R} \times \mathbb{K})
$$

such that $\sigma_{n} \rightarrow \sigma$ in $L_{\mu_{\alpha}}^{p}(\mathbb{R} \times \mathbb{K})$ as $n \rightarrow \infty$. Then by Theorem 3.5

$$
\left\|\mathcal{L}_{h, k}\left(\sigma_{n}\right)-\mathcal{L}_{h, k}(\sigma)\right\|_{S_{\infty}} \leq\left(C_{h} C_{k}\right)^{\frac{p-1}{2 p}}\left\|\sigma_{n}-\sigma\right\|_{L_{\mu_{\alpha}}^{p}(\mathbb{R} \times \mathbb{K})} .
$$

Hence $\mathcal{L}_{h, k}\left(\sigma_{n}\right) \rightarrow \mathcal{L}_{h, k}(\sigma)$ in $S_{\infty}$ as $n \rightarrow \infty$. On the other hand, as by Proposition $3.6 \mathcal{L}_{h, k}\left(\sigma_{n}\right)$ is in $S_{2}$ and hence compact, it follows that $\mathcal{L}_{h, k}(\sigma)$ is compact.

Theorem 3.8. Let $\sigma$ be in $L_{\mu_{\alpha}}^{1}(\mathbb{R} \times \mathbb{K})$. Then $\mathcal{L}_{h, k}(\sigma): L_{\alpha}^{2}(\mathbb{K}) \rightarrow L_{\alpha}^{2}(\mathbb{K})$ is in $S_{1}$ and we have

$$
\frac{2}{C_{h}+C_{k}}\|\widetilde{\sigma}\|_{L_{\mu_{\alpha}}^{1}(\mathbb{R} \times \mathbb{K})} \leq\left\|\mathcal{L}_{h, k}(\sigma)\right\|_{S_{1}} \leq\|\sigma\|_{L_{\mu_{\alpha}}^{1}(\mathbb{R} \times \mathbb{K})},
$$

where $\widetilde{\sigma}$ is given by

$$
\forall(a, x, t) \in \mathbb{R} \times \mathbb{K}, \quad \tilde{\sigma}(a, x, t)=\left\langle\mathcal{L}_{h, k}(\sigma) h_{a, x, t}, k_{a, x, t}\right\rangle_{L_{\alpha}^{2}(\mathbb{K})} .
$$

Proof. Since $\sigma$ is in $L_{\mu_{\alpha}}^{1}(\mathbb{R} \times \mathbb{K})$, by Proposition $3.6 \mathcal{L}_{h, k}(\sigma)$ is in $S_{2}$; then from the canonical form for compact operators given in [35, Theorem 2.2], there exists an orthonormal basis $\left\{\phi_{j}, j=1,2, \ldots\right\}$ for the orthogonal complement of the kernel of the operator $\mathcal{L}_{h, k}(\sigma)$, consisting of eigenvectors of $\left|\mathcal{L}_{h, k}(\sigma)\right|$ and an orthonormal set $\left\{u_{j}, j=1,2, \ldots\right\}$ in $L_{\alpha}^{2}(\mathbb{K})$ such that

$$
\mathcal{L}_{h, k}(\sigma)(f)=\sum_{j=1}^{\infty} s_{j}\left\langle f, \phi_{j}\right\rangle_{L_{\alpha}^{2}(\mathbb{K})} u_{j},
$$

where $s_{j}, j=1,2, \ldots$, are the positive singular values of $\mathcal{L}_{h, k}(\sigma)$ corresponding to $\phi_{j}$. Then, we get

$$
\left\|\mathcal{L}_{h, k}(\sigma)\right\|_{S_{1}}=\sum_{j=1}^{\infty} s_{j}=\sum_{j=1}^{\infty}\left\langle\mathcal{L}_{h, k}(\sigma)\left(\phi_{j}\right), u_{j}\right\rangle_{L_{\alpha}^{2}(\mathbb{K})}
$$


Thus, by Fubini's theorem, Cauchy-Schwarz's inequality, Bessel's inequality, and relations 2.8 and $(2.6)$, we get

$$
\begin{aligned}
\left\|\mathcal{L}_{h, k}(\sigma)\right\|_{S_{1}} & =\sum_{j=1}^{\infty}\left\langle\mathcal{L}_{h, k}(\sigma)\left(\phi_{j}\right), u_{j}\right\rangle_{L_{\alpha}^{2}(\mathbb{K})} \\
= & \sum_{j=1}^{\infty} \int_{\mathbb{R} \times \mathbb{K}} \sigma(a, x, t) \Phi_{h}^{\alpha}\left(\phi_{j}\right)(a, x, t) \overline{\Phi_{k}^{\alpha}\left(u_{j}\right)(a, x, t)} d \mu_{\alpha}(a, x, t) \\
\leq & \int_{\mathbb{R} \times \mathbb{K}}|\sigma(a, x, t)|\left(\sum_{j=1}^{\infty}\left|\Phi_{h}^{\alpha}\left(\phi_{j}\right)(a, x, t)\right|^{2}\right)^{\frac{1}{2}} \\
& \times\left(\sum_{j=1}^{\infty}\left|\Phi_{k}^{\alpha}\left(u_{j}\right)(a, x, t)\right|^{2}\right)^{\frac{1}{2}} d \mu_{\alpha}(a, x, t) \\
\leq & \int_{\mathbb{R} \times \mathbb{K}}|\sigma(a, x, t)|\left\|h_{a, x, t}\right\|_{L_{\alpha}^{2}(\mathbb{K})}\left\|k_{a, x, t}\right\|_{L_{\alpha}^{2}(\mathbb{K})} d \mu_{\alpha}(a, x, t) \\
\leq & \|\sigma\|_{L_{\mu_{\alpha}}^{1}(\mathbb{R} \times \mathbb{K}) .}
\end{aligned}
$$

Thus

$$
\left\|\mathcal{L}_{h, k}(\sigma)\right\|_{S_{1}} \leq\|\sigma\|_{L_{\mu_{\alpha}}^{1}(\mathbb{R} \times \mathbb{K})} .
$$

We now prove that $\mathcal{L}_{h, k}(\sigma)$ satisfies the first inequality of $(3.9)$. It is easy to see that $\widetilde{\sigma}$ belongs to $L_{\alpha}^{1}(\mathbb{K})$, and using formula 3.10 we get

$$
\begin{aligned}
|\widetilde{\sigma}(a, x, t)| & =\left|\left\langle\mathcal{L}_{h, k}(\sigma)\left(h_{a, x, t}\right), k_{a, x, t}\right\rangle_{L_{\alpha}^{2}(\mathbb{K})}\right| \\
& =\left|\sum_{j=1}^{\infty} s_{j}\left\langle h_{a, x, t}, \phi_{j}\right\rangle_{L_{\alpha}^{2}(\mathbb{K})}\left\langle u_{j}, k_{a, x, t}\right\rangle_{L_{\alpha}^{2}(\mathbb{K})}\right| \\
& \leq \frac{1}{2} \sum_{j=1}^{\infty} s_{j}\left(\left|\left\langle h_{a, x, t}, \phi_{j}\right\rangle_{L_{\alpha}^{2}(\mathbb{K})}\right|^{2}+\left|\left\langle k_{a, x, t}, u_{j}\right\rangle_{L_{\alpha}^{2}(\mathbb{K})}\right|^{2}\right) .
\end{aligned}
$$

Then from Fubini's theorem, we obtain

$$
\begin{aligned}
\int_{\mathbb{R} \times \mathbb{K}}|\widetilde{\sigma}(a, x, t)| d \mu_{\alpha}(a, x, t) \leq & \frac{1}{2} \sum_{j=1}^{\infty} s_{j}\left(\int_{\mathbb{R} \times \mathbb{K}}\left|\left\langle h_{a, x, t}, \phi_{j}\right\rangle_{L_{\alpha}^{2}(\mathbb{K})}\right|^{2} d \mu_{\alpha}(a, x, t)\right. \\
& \left.+\int_{\mathbb{R} \times \mathbb{K}}\left|\left\langle k_{a, x, t}, u_{j}\right\rangle_{L_{\alpha}^{2}(\mathbb{K})}\right|^{2} d \mu_{\alpha}(a, x, t)\right) .
\end{aligned}
$$

Thus using Plancherel's formula for $\Phi_{h}^{\alpha}, \Phi_{k}^{\alpha}$ we get

$$
\int_{\mathbb{R} \times \mathbb{K}}|\widetilde{\sigma}(a, x, t)| d \mu_{\alpha}(a, x, t) \leq \frac{C_{h}+C_{k}}{2} \sum_{j=1}^{\infty} s_{j}=\frac{C_{h}+C_{k}}{2}\left\|\mathcal{L}_{h, k}(\sigma)\right\|_{S_{1}} .
$$

The proof is complete. 
Corollary 3.9. For $\sigma$ in $L_{\mu_{\alpha}}^{1}(\mathbb{R} \times \mathbb{K})$, we have the following trace formula:

$$
\operatorname{tr}\left(\mathcal{L}_{h, k}(\sigma)\right)=\int_{\mathbb{R} \times \mathbb{K}} \sigma(a, x, t)\left\langle k_{a, x, t}, h_{a, x, t}\right\rangle_{L_{\alpha}^{2}(\mathbb{K})} d \mu_{\alpha}(a, x, t) .
$$

Proof. Let $\left\{\phi_{j}, j=1,2, \ldots\right\}$ be an orthonormal basis for $L_{\alpha}^{2}(\mathbb{K})$. From Theorem 3.8 , the localization operator $\mathcal{L}_{h, k}(\sigma)$ belongs to $S_{1}$; then by the definition of trace given by relation (2.3), Fubini's theorem, and Parseval's identity, we have

$$
\begin{aligned}
\operatorname{tr}\left(\mathcal{L}_{h, k}(\sigma)\right) & =\sum_{j=1}^{\infty}\left\langle\mathcal{L}_{h, k}(\sigma)\left(\phi_{j}\right), \phi_{j}\right\rangle_{L_{\alpha}^{2}(\mathbb{K})} \\
& =\sum_{j=1}^{\infty} \int_{\mathbb{R} \times \mathbb{K}} \sigma(a, x, t)\left\langle\phi_{j}, h_{a, x, t}\right\rangle_{L_{\alpha}^{2}(\mathbb{K})} \overline{\left\langle\phi_{j}, k_{a, x, t}\right\rangle_{L_{\alpha}^{2}(\mathbb{K})}} d \mu_{\alpha}(a, x, t) \\
& =\int_{\mathbb{R} \times \mathbb{K}} \sigma(a, x, t) \sum_{j=1}^{\infty}\left\langle\phi_{j}, h_{a, x, t}\right\rangle_{L_{\alpha}^{2}(\mathbb{K})} \overline{\left\langle\phi_{j}, k_{a, x, t}\right\rangle_{L_{\alpha}^{2}(\mathbb{K})}} d \mu_{\alpha}(a, x, t) \\
& =\int_{\mathbb{R} \times \mathbb{K}} \sigma(a, x, t)\left\langle k_{a, x, t}, h_{a, x, t}\right\rangle_{L_{\alpha}^{2}(\mathbb{K})} d \mu_{\alpha}(a, x, t),
\end{aligned}
$$

and the proof is complete.

In the following we give the main result of this subsection.

Corollary 3.10. Let $\sigma$ be in $L_{\mu_{\alpha}}^{p}(\mathbb{R} \times \mathbb{K}), 1 \leq p \leq \infty$. Then, the localization operator

is in $S_{p}$ and we have

$$
\mathcal{L}_{h, k}(\sigma): L_{\alpha}^{2}(\mathbb{K}) \rightarrow L_{\alpha}^{2}(\mathbb{K})
$$

$$
\left\|\mathcal{L}_{h, k}(\sigma)\right\|_{S_{p}} \leq\left(C_{h} C_{k}\right)^{\frac{p-1}{2 p}}\|\sigma\|_{L_{\mu_{\alpha}}^{p}(\mathbb{R} \times \mathbb{K})} .
$$

Proof. The result follows from Proposition 3.4. Theorem 3.8 and by interpolation (see [35, Theorem 2.10 and Theorem 2.11]).

Remark 3.11. If $h=k$ and $\sigma$ is a real valued and nonnegative function in $L_{\mu_{\alpha}}^{1}(\mathbb{R} \times \mathbb{K})$, then $\mathcal{L}_{h, k}(\sigma): L_{\alpha}^{2}(\mathbb{K}) \rightarrow L_{\alpha}^{2}(\mathbb{K})$ is a positive operator. So, by 2.4 and Corollary 3.9

$$
\left\|\mathcal{L}_{h, k}(\sigma)\right\|_{S_{1}}=\int_{\mathbb{R} \times \mathbb{K}} \sigma(a, x, t)\left\|h_{a, x, t}\right\|_{L_{\alpha}^{2}(\mathbb{K})}^{2} d \mu_{\alpha}(a, x, t) .
$$

Now we state a result concerning the trace of products of localization operators.

Corollary 3.12. Let $\sigma_{1}$ and $\sigma_{2}$ be any real-valued and non-negative functions in $L_{\mu_{\alpha}}^{1}(\mathbb{R} \times \mathbb{K})$. We assume that $h=k$ and that $h$ is a function in $L_{\alpha}^{2}(\mathbb{K})$ such that $\|h\|_{L_{\alpha}^{2}(\mathbb{K})}=1$. Then, the localization operators $\mathcal{L}_{h, k}\left(\sigma_{1}\right), \mathcal{L}_{h, k}\left(\sigma_{2}\right)$ are positive trace class operators and, for any natural number $n$,

$$
\begin{aligned}
\left\|\left(\mathcal{L}_{h, k}\left(\sigma_{1}\right) \mathcal{L}_{h, k}\left(\sigma_{2}\right)\right)^{n}\right\|_{S_{1}} & =\operatorname{tr}\left(\mathcal{L}_{h, k}\left(\sigma_{1}\right) \mathcal{L}_{h, k}\left(\sigma_{2}\right)\right)^{n} \\
& \leq\left(\operatorname{tr}\left(\mathcal{L}_{h, k}\left(\sigma_{1}\right)\right)\right)^{n}\left(\operatorname{tr}\left(\mathcal{L}_{h, k}\left(\sigma_{2}\right)\right)\right)^{n} \\
& =\left\|\mathcal{L}_{h, k}\left(\sigma_{1}\right)\right\|_{S_{1}}^{n}\left\|\mathcal{L}_{h, k}\left(\sigma_{2}\right)\right\|_{S_{1}}^{n} .
\end{aligned}
$$


Proof. By Theorem 1 in Liu's paper [22] we know that if $A$ and $B$ are in the trace class $S_{1}$ and are positive operators, then

$$
\forall n \in \mathbb{N}, \quad \operatorname{tr}(A B)^{n} \leq(\operatorname{tr}(A))^{n}(\operatorname{tr}(B))^{n} .
$$

So, if we take $A=\mathcal{L}_{h, k}\left(\sigma_{1}\right), B=\mathcal{L}_{h, k}\left(\sigma_{2}\right)$, and we invoke the previous remark, the desired result is obtained and the proof is complete.

\section{4. $L_{\alpha}^{p}$ BOUNDEDNESS AND COMPACTNESS OF $\mathcal{L}_{h, k}(\sigma)$}

In this section we will derive a host of sufficient conditions for the boundedness and compactness of the localization operators $\mathcal{L}_{h, k}(\sigma)$ on $L_{\alpha}^{p}(\mathbb{K}), 1 \leq p \leq \infty$, in terms of properties of the symbol $\sigma$ and the windows $h$ and $k$.

4.1. Boundedness of $\mathcal{L}_{h, k}(\sigma)$ for symbols in $L_{\alpha}^{p}(\mathbb{K})$. For $1 \leq p \leq \infty$, let $\sigma \in L_{\mu_{\alpha}}^{1}(\mathbb{R} \times \mathbb{K}), k \in L_{\alpha}^{p}(\mathbb{K})$, and $h \in L_{\alpha}^{p^{\prime}}(\mathbb{K})$. We are going to show that $\mathcal{L}_{h, k}(\sigma)$ is a bounded operator on $L_{\alpha}^{p}(\mathbb{K})$. Let us start with the following propositions.

Proposition 4.1. Let $\sigma$ be in $L_{\mu_{\alpha}}^{1}(\mathbb{R} \times \mathbb{K}), h \in L_{\alpha}^{\infty}(\mathbb{K})$, and $k \in L_{\alpha}^{1}(\mathbb{K})$. Then the localization operator $\mathcal{L}_{h, k}(\sigma): L_{\alpha}^{1}(\mathbb{K}) \rightarrow L_{\alpha}^{1}(\mathbb{K})$ is a bounded linear operator and we have

$$
\left\|\mathcal{L}_{h, k}(\sigma)\right\|_{B\left(L_{\alpha}^{1}(\mathbb{K})\right)} \leq\|h\|_{L_{\alpha}^{\infty}(\mathbb{K})}\|k\|_{L_{\alpha}^{1}(\mathbb{K})}\|\sigma\|_{L_{\mu_{\alpha}}^{1}(\mathbb{R} \times \mathbb{K})} .
$$

Proof. For every function $f$ in $L_{\alpha}^{1}(\mathbb{K})$, from Fubini's theorem and the relations (3.1), 2.11), and (2.7), we have

$$
\begin{aligned}
\left\|\mathcal{L}_{h, k}(\sigma)(f)\right\|_{L_{\alpha}^{1}(\mathbb{K})} & \leq \int_{\mathbb{K}} \int_{\mathbb{R} \times \mathbb{K}}|\sigma(a, x, t)|\left|\Phi_{h}^{\alpha}(f)(a, x, t)\right|\left|k_{a, x, t}(s, y)\right| d \mu_{\alpha}(a, x, t) d \nu_{\alpha}(s, y) \\
& \leq\|f\|_{L_{\alpha}^{1}(\mathbb{K})}\|h\|_{L_{\alpha}^{\infty}(\mathbb{K})}\|k\|_{L_{\alpha}^{1}(\mathbb{K})}\|\sigma\|_{L_{\mu_{\alpha}}^{1}(\mathbb{R} \times \mathbb{K})} .
\end{aligned}
$$

Thus,

$$
\left\|\mathcal{L}_{h, k}(\sigma)\right\|_{B\left(L_{\alpha}^{1}(\mathbb{K})\right)} \leq\|h\|_{L_{\alpha}^{\infty}(\mathbb{K})}\|k\|_{L_{\alpha}^{1}(\mathbb{K})}\|\sigma\|_{L_{\mu_{\alpha}}^{1}(\mathbb{R} \times \mathbb{K})}
$$

Proposition 4.2. Let $\sigma$ be in $L_{\mu_{\alpha}}^{1}(\mathbb{R} \times \mathbb{K}), h \in L_{\alpha}^{1}(\mathbb{K})$, and $k \in L_{\alpha}^{\infty}(\mathbb{K})$. Then the localization operator $\mathcal{L}_{h, k}(\sigma): L_{\alpha}^{\infty}(\mathbb{K}) \rightarrow L_{\alpha}^{\infty}(\mathbb{K})$ is a bounded linear operator and we have

$$
\left\|\mathcal{L}_{h, k}(\sigma)\right\|_{B\left(L_{\alpha}^{\infty}(\mathbb{K})\right)} \leq\|h\|_{L_{\alpha}^{1}(\mathbb{K})}\|k\|_{L_{\alpha}^{\infty}(\mathbb{K})}\|\sigma\|_{L_{\mu_{\alpha}}^{1}(\mathbb{R} \times \mathbb{K})} .
$$

Proof. Let $f$ be in $L_{\alpha}^{\infty}(\mathbb{K})$. As above, from Fubini's theorem and the relations (3.1), 2.11), and (2.7), we have

$$
\begin{aligned}
\left|\mathcal{L}_{h, k}(\sigma)(f)(s, y)\right| & \leq \int_{\mathbb{R} \times \mathbb{K}}|\sigma(a, x, t)|\left|\Phi_{h}^{\alpha}(f)(a, x, t)\right|\left|k_{a, x, t}(s, y)\right| d \mu_{\alpha}(a, x, t) \\
& \leq\|f\|_{L_{\alpha}^{\infty}(\mathbb{K})}\|h\|_{L_{\alpha}^{1}(\mathbb{K})}\|k\|_{L_{\alpha}^{\infty}(\mathbb{K})}\|\sigma\|_{L_{\mu_{\alpha}}^{1}(\mathbb{R} \times \mathbb{K})}, \quad \forall(s, y) \in \mathbb{K} .
\end{aligned}
$$

Thus,

$$
\left\|\mathcal{L}_{h, k}(\sigma)\right\|_{B\left(L_{\alpha}^{\infty}(\mathbb{K})\right)} \leq\|h\|_{L_{\alpha}^{1}(\mathbb{K})}\|k\|_{L_{\alpha}^{\infty}(\mathbb{K})}\|\sigma\|_{L_{\mu_{\alpha}}^{1}(\mathbb{R} \times \mathbb{K})} .
$$

Remark 4.3. We note that Proposition 4.2 is also a corollary of Proposition 4.1 since the adjoint of $\mathcal{L}_{k, h}(\bar{\sigma}): L_{\alpha}^{1}(\mathbb{K}) \rightarrow L_{\alpha}^{1}(\mathbb{K})$ is $\mathcal{L}_{h, k}(\sigma): L_{\alpha}^{\infty}(\mathbb{K}) \rightarrow L_{\alpha}^{\infty}(\mathbb{K})$.

Using an interpolation of Propositions 4.1 and 4.2 , we get the following result. 
Theorem 4.4. Let $h$ and $k$ be functions in $L_{\alpha}^{1}(\mathbb{K}) \cap L_{\alpha}^{\infty}(\mathbb{K})$. Then for all $\sigma$ in $L_{\mu_{\alpha}}^{1}(\mathbb{R} \times \mathbb{K})$, there exists a unique bounded linear operator

$$
\mathcal{L}_{h, k}(\sigma): L_{\alpha}^{p}(\mathbb{K}) \rightarrow L_{\alpha}^{p}(\mathbb{K}), \quad 1 \leq p \leq \infty,
$$

such that

$$
\left\|\mathcal{L}_{h, k}(\sigma)\right\|_{B\left(L_{\alpha}^{p}(\mathbb{K})\right)} \leq\|h\|_{L_{\alpha}^{1}(\mathbb{K})}^{\frac{1}{p^{\prime}}}\|k\|_{L_{\alpha}^{1}(\mathbb{K})}^{\frac{1}{p}}\|h\|_{L_{\alpha}^{\infty}(\mathbb{K})}^{\frac{1}{p}}\|k\|_{L_{\alpha}^{\infty}(\mathbb{K})}^{\frac{1}{p^{\prime}}}\|\sigma\|_{L_{\mu_{\alpha}}^{1}(\mathbb{R} \times \mathbb{K})} .
$$

With a Schur technique, we can obtain an $L_{\alpha}^{p}$-boundedness result as in the previous theorem, but the estimate for the norm $\left\|\mathcal{L}_{h, k}(\sigma)\right\|_{B\left(L_{\alpha}^{p}(\mathbb{K})\right)}$ is cruder.

Theorem 4.5. Let $\sigma$ be in $L_{\mu_{\alpha}}^{1}(\mathbb{R} \times \mathbb{K})$, $h$ and $k$ in $L_{\alpha}^{1}(\mathbb{K}) \cap L_{\alpha}^{\infty}(\mathbb{K})$. Then there exists a unique bounded linear operator

$$
\mathcal{L}_{h, k}(\sigma): L_{\alpha}^{p}(\mathbb{K}) \rightarrow L_{\alpha}^{p}(\mathbb{K}), \quad 1 \leq p \leq \infty,
$$

such that

$$
\left\|\mathcal{L}_{h, k}(\sigma)\right\|_{B\left(L_{\alpha}^{p}(\mathbb{K})\right)} \leq \max \left(\|h\|_{L_{\alpha}^{1}(\mathbb{K})}\|k\|_{L_{\alpha}^{\infty}(\mathbb{K})},\|h\|_{L_{\alpha}^{\infty}(\mathbb{K})}\|k\|_{L_{\alpha}^{1}(\mathbb{K})}\right)\|\sigma\|_{L_{\mu_{\alpha}}^{1}(\mathbb{R} \times \mathbb{K})} .
$$

Proof. Let $\mathcal{N}$ be the function defined on $\mathbb{K} \times \mathbb{K}$ by

$$
\mathcal{N}(s, y ; t, z)=\int_{\mathbb{R} \times \mathbb{K}} \sigma(a, x, t) \overline{h_{a, x, t}(t, z)} k_{a, x, t}(s, y) d \mu_{\alpha}(a, x, t) .
$$

We have

$$
\mathcal{L}_{h, k}(\sigma)(f)(s, y)=\int_{\mathbb{K}} \mathcal{N}(s, y ; t, z) f(t, z) d \nu_{\alpha}(t, z) .
$$

By simple calculations, it is easy to see that

$$
\int_{\mathbb{K}}|\mathcal{N}(s, y ; t, z)| d \nu_{\alpha}(s, y) \leq\|h\|_{L_{\alpha}^{\infty}(\mathbb{K})}\|k\|_{L_{\alpha}^{1}(\mathbb{K})}\|\sigma\|_{L_{\mu_{\alpha}}^{1}(\mathbb{R} \times \mathbb{K})}, \quad(t, z) \in \mathbb{K},
$$

and

$$
\int_{\mathbb{K}}|\mathcal{N}(s, y ; t, z)| d \nu_{\alpha}(t, z) \leq\|h\|_{L_{\alpha}^{1}(\mathbb{K})}\|k\|_{L_{\alpha}^{\infty}(\mathbb{K})}\|\sigma\|_{L_{\mu_{\alpha}}^{1}(\mathbb{R} \times \mathbb{K})}, \quad(s, y) \in \mathbb{K} .
$$

Thus by Schur's lemma (see [15]), we can conclude that $\mathcal{L}_{h, k}(\sigma): L_{\alpha}^{p}(\mathbb{K}) \rightarrow L_{\alpha}^{p}(\mathbb{K})$ is a bounded linear operator for $1 \leq p \leq \infty$, and we have

$$
\left\|\mathcal{L}_{h, k}(\sigma)\right\|_{B\left(L_{\alpha}^{p}(\mathbb{K})\right)} \leq \max \left(\|h\|_{L_{\alpha}^{1}(\mathbb{K})}\|k\|_{L_{\alpha}^{\infty}(\mathbb{K})},\|h\|_{L_{\alpha}^{\infty}(\mathbb{K})}\|k\|_{L_{\alpha}^{1}(\mathbb{K})}\right)\|\sigma\|_{L_{\mu_{\alpha}}^{1}(\mathbb{R} \times \mathbb{K})} .
$$

Remark 4.6. The previous theorem tells us that the unique bounded linear operator on $L_{\alpha}^{p}(\mathbb{K}), 1 \leq p \leq \infty$, obtained by interpolation in Theorem 4.4 is in fact the integral operator on $L_{\alpha}^{p}(\mathbb{K})$ with kernel $\mathcal{N}$ given by 4.1 .

We can give another version of the $L_{\alpha}^{p}$-boundedness. We first generalize and improve Proposition 4.2 .

Proposition 4.7. Let $\sigma$ be in $L_{\mu_{\alpha}}^{1}(\mathbb{R} \times \mathbb{K}), k \in L_{\alpha}^{p}(\mathbb{K})$, and $h \in L_{\alpha}^{p^{\prime}}(\mathbb{K})$, for $1<p \leq \infty$. Then the localization operator $\mathcal{L}_{h, k}(\sigma): L_{\alpha}^{p}(\mathbb{K}) \rightarrow L_{\alpha}^{p}(\mathbb{K})$ is a bounded linear operator, and we have

$$
\left\|\mathcal{L}_{h, k}(\sigma)\right\|_{B\left(L_{\alpha}^{p}(\mathbb{K})\right)} \leq\|h\|_{L_{\alpha}^{p^{\prime}}(\mathbb{K})}\|k\|_{L_{\alpha}^{p}(\mathbb{K})}\|\sigma\|_{L_{\mu_{\alpha}}^{1}(\mathbb{R} \times \mathbb{K})}
$$


Proof. For any $f \in L_{\alpha}^{p}(\mathbb{K})$, consider the linear functional

$$
\begin{aligned}
\mathcal{I}_{f}: L_{\alpha}^{p^{\prime}}(\mathbb{K}) & \rightarrow \mathbb{C} \\
g & \mapsto\left\langle g, \mathcal{L}_{h, k}(\sigma)(f)\right\rangle_{L_{\alpha}^{2}(\mathbb{K})}
\end{aligned}
$$

By 3.2 , we have

$$
\left|\left\langle\mathcal{L}_{h, k}(\sigma)(f), g\right\rangle_{L_{\alpha}^{2}(\mathbb{K})}\right| \leq \int_{\mathbb{R} \times \mathbb{K}}|\sigma(a, x, t)|\left|\Phi_{h}^{\alpha}(f)(a, x, t)\right|\left|\overline{\Phi_{k}^{\alpha}(g)(a, x, t)}\right| d \mu_{\alpha}(a, x, t) .
$$

Using Fubini's theorem and the relation 2.11, we get

$$
\left|\left\langle\mathcal{L}_{h, k}(\sigma)(f), g\right\rangle_{L_{\alpha}^{2}(\mathbb{K})}\right| \leq\|h\|_{L_{\alpha}^{p^{\prime}(\mathbb{K})}}\|k\|_{L_{\alpha}^{p}(\mathbb{K})}\|f\|_{L_{\alpha}^{p}(\mathbb{K})}\|g\|_{L_{\alpha}^{p^{\prime}}(\mathbb{K})}\|\sigma\|_{L_{\mu_{\alpha}}^{1}(\mathbb{R} \times \mathbb{K})} .
$$

Thus, the operator $\mathcal{I}_{f}$ is a continuous linear functional on $L_{\alpha}^{p^{\prime}}(\mathbb{K})$, and the operator norm satisfies

$$
\left\|\mathcal{I}_{f}\right\|_{B\left(L_{\alpha}^{\left.p^{\prime}(\mathbb{K})\right)}\right.} \leq\|h\|_{L_{\alpha}^{p^{\prime}(\mathbb{K})}}\|k\|_{L_{\alpha}^{p}(\mathbb{K})}\|f\|_{L_{\alpha}^{p}(\mathbb{K})}\|\sigma\|_{L_{\mu_{\alpha}}^{1}(\mathbb{R} \times \mathbb{K})} .
$$

As $\mathcal{I}_{f}(g)=\left\langle g, \mathcal{L}_{h, k}(\sigma)(f)\right\rangle_{L_{\alpha}^{2}(\mathbb{K})}$, by the Riesz representation theorem we have

$$
\left\|\mathcal{L}_{h, k}(\sigma)(f)\right\|_{L_{\alpha}^{p}(\mathbb{K})}=\left\|\mathcal{I}_{f}\right\|_{B\left(L_{\alpha}^{p^{\prime}}(\mathbb{K})\right)} \leq\|h\|_{L_{\alpha}^{p^{\prime}(\mathbb{K})}}\|k\|_{L_{\alpha}^{p}(\mathbb{K})}\|f\|_{L_{\alpha}^{p}(\mathbb{K})}\|\sigma\|_{L_{\mu_{\alpha}}^{1}(\mathbb{R} \times \mathbb{K})},
$$

which establishes the proposition.

Combining Proposition 4.1 and Proposition 4.7. we have the following theorem.

Theorem 4.8. Let $\sigma$ be in $L_{\mu_{\alpha}}^{1}(\mathbb{R} \times \mathbb{K}), k \in L_{\alpha}^{p}(\mathbb{K}), h \in L_{\alpha}^{p^{\prime}}(\mathbb{K}), 1 \leq p \leq \infty$. Then the localization operator $\mathcal{L}_{h, k}(\sigma): L_{\alpha}^{p}(\mathbb{K}) \rightarrow L_{\alpha}^{p}(\mathbb{K})$ is a bounded linear operator, and we have

$$
\left\|\mathcal{L}_{h, k}(\sigma)\right\|_{B\left(L_{\alpha}^{p}(\mathbb{K})\right)} \leq\|h\|_{L_{\alpha}^{p^{\prime}(\mathbb{K})}}\|k\|_{L_{\alpha}^{p}(\mathbb{K})}\|\sigma\|_{L_{\mu_{\alpha}}^{1}(\mathbb{R} \times \mathbb{K})} .
$$

We can now state and prove the main results in this subsection.

Theorem 4.9. Let $\sigma$ be in $L_{\mu_{\alpha}}^{r}(\mathbb{R} \times \mathbb{K}), r \in[1,2]$, and $h, k \in L_{\alpha}^{1}(\mathbb{K}) \cap L_{\alpha}^{2}(\mathbb{K}) \cap$ $L_{\alpha}^{\infty}(\mathbb{K})$. Then there exists a unique bounded linear operator $\mathcal{L}_{h, k}(\sigma): L_{\alpha}^{p}(\mathbb{K}) \rightarrow$ $L_{\alpha}^{p}(\mathbb{K})$ for all $p \in\left[r, r^{\prime}\right]$, and we have

$$
\left\|\mathcal{L}_{h, k}(\sigma)\right\|_{B\left(L_{\alpha}^{p}(\mathbb{K})\right)} \leq C_{1}^{t} C_{2}^{1-t}\|\sigma\|_{L_{\mu_{\alpha}}^{r}(\mathbb{R} \times \mathbb{K})}
$$

where

$$
\begin{aligned}
& C_{1}=\left(\|h\|_{L_{\alpha}^{\infty}(\mathbb{K})}\|k\|_{L_{\alpha}^{1}(\mathbb{K})}\right)^{\frac{2}{r}-1}\left(\sqrt{C_{h} C_{k}}\|h\|_{L_{\alpha}^{2}(\mathbb{K})}\|k\|_{L_{\alpha}^{2}(\mathbb{K})}\right)^{\frac{1}{r^{\prime}}}, \\
& C_{2}=\left(\|h\|_{L_{\alpha}^{1}(\mathbb{K})}\|k\|_{L_{\alpha}^{\infty}(\mathbb{K})}\right)^{\frac{2}{r}-1}\left(\sqrt{C_{h} C_{k}}\|h\|_{L_{\alpha}^{2}(\mathbb{K})}\|k\|_{L_{\alpha}^{2}(\mathbb{K})}\right)^{\frac{1}{r^{\prime}}},
\end{aligned}
$$

with

$$
\frac{t}{r}+\frac{1-t}{r^{\prime}}=\frac{1}{p}
$$


Proof. Consider the linear functional

$$
\begin{aligned}
\mathcal{I}:\left(L_{\mu_{\alpha}}^{1}(\mathbb{R} \times \mathbb{K}) \cap L_{\mu_{\alpha}}^{2}(\mathbb{R} \times \mathbb{K})\right) \times\left(L_{\alpha}^{1}(\mathbb{K}) \cap L_{\alpha}^{2}(\mathbb{K})\right) & \rightarrow L_{\alpha}^{1}(\mathbb{K}) \cap L_{\alpha}^{2}(\mathbb{K}) \\
(\sigma, f) & \mapsto \mathcal{L}_{h, k}(\sigma)(f) .
\end{aligned}
$$

By Proposition 4.1 and Theorem 3.5, we have

$$
\|\mathcal{I}(\sigma, f)\|_{L_{\alpha}^{1}(\mathbb{K})} \leq\|h\|_{L_{\alpha}^{\infty}(\mathbb{K})}\|k\|_{L_{\alpha}^{1}(\mathbb{K})}\|f\|_{L_{\alpha}^{1}(\mathbb{K})}\|\sigma\|_{L_{\mu_{\alpha}}^{1}(\mathbb{R} \times \mathbb{K})}
$$

and

$$
\|\mathcal{I}(\sigma, f)\|_{L_{\alpha}^{2}(\mathbb{K})} \leq\left(\sqrt{C_{h} C_{k}}\|h\|_{L_{\alpha}^{2}(\mathbb{K})}\|k\|_{L_{\alpha}^{2}(\mathbb{K})}\right)^{\frac{1}{2}}\|f\|_{L_{\alpha}^{2}(\mathbb{K})}\|\sigma\|_{L_{\mu_{\alpha}}^{2}(\mathbb{R} \times \mathbb{K})} .
$$

Therefore, by 4.2, 4.3), and the multi-linear interpolation theory (see [5, Section 10.1] for reference), we get a unique bounded linear operator

$$
\mathcal{I}: L_{\mu_{\alpha}}^{r}(\mathbb{R} \times \mathbb{K}) \times L_{\alpha}^{r}(\mathbb{K}) \rightarrow L_{\alpha}^{r}(\mathbb{K})
$$

such that

$$
\|\mathcal{I}(\sigma, f)\|_{L_{\alpha}^{r}(\mathbb{K})} \leq C_{1}\|f\|_{L_{\alpha}^{r}(\mathbb{K})}\|\sigma\|_{L_{\mu_{\alpha}}^{r}(\mathbb{R} \times \mathbb{K})}
$$

where

$$
C_{1}=\left(\|h\|_{L_{\alpha}^{\infty}(\mathbb{K})}\|k\|_{L_{\alpha}^{1}(\mathbb{K})}\right)^{\theta}\left(\sqrt{C_{h} C_{k}}\|h\|_{L_{\alpha}^{2}(\mathbb{K})}\|k\|_{L_{\alpha}^{2}(\mathbb{K})}\right)^{\frac{1-\theta}{2}}
$$

and

$$
\frac{\theta}{1}+\frac{1-\theta}{2}=\frac{1}{r}
$$

By the definition of $\mathcal{I}$, we have

$$
\begin{aligned}
\left\|\mathcal{L}_{h, k}(\sigma)\right\|_{B\left(L_{\alpha}^{r}(\mathbb{K})\right) \leq} \leq & \left.\|h\|_{L_{\alpha}^{\infty}(\mathbb{K})}\|k\|_{L_{\alpha}^{1}(\mathbb{K})}\right)^{\frac{2}{r}-1} \\
& \times\left(\sqrt{C_{h} C_{k}}\|h\|_{L_{\alpha}^{2}(\mathbb{K})}\|k\|_{L_{\alpha}^{2}(\mathbb{K})}\right)^{\frac{1}{r^{\prime}}}\|\sigma\|_{L_{\mu_{\alpha}}^{r}(\mathbb{R} \times \mathbb{K})} .
\end{aligned}
$$

As the adjoint of $\mathcal{L}_{h, k}(\sigma)$ is $\mathcal{L}_{k, h}(\bar{\sigma}), \mathcal{L}_{h, k}(\sigma)$ is a bounded linear map on $L_{\alpha}^{r^{\prime}}(\hat{\mathbb{K}})$ with its operator norm satisfying

$$
\left\|\mathcal{L}_{h, k}(\sigma)\right\|_{B\left(L_{\alpha}^{r^{\prime}}(\mathbb{K})\right)}=\left\|\mathcal{L}_{k, h}(\bar{\sigma})\right\|_{B\left(L_{\alpha}^{r}(\mathbb{K})\right)} \leq C_{2}\|\sigma\|_{L_{\mu_{\alpha}}^{r}(\mathbb{R} \times \mathbb{K})},
$$

where

$$
C_{2}=\left(\|h\|_{L_{\alpha}^{1}(\mathbb{K})}\|k\|_{L_{\alpha}^{\infty}(\mathbb{K})}\right)^{\frac{2}{r}-1}\left(\sqrt{C_{h} C_{k}}\|h\|_{L_{\alpha}^{2}(\mathbb{K})}\|k\|_{L_{\alpha}^{2}(\mathbb{K})}\right)^{\frac{1}{r^{\prime}}} .
$$

Using an interpolation of (4.4) and 4.5, we have that, for any $p \in\left[r, r^{\prime}\right]$,

$$
\left\|\mathcal{L}_{h, k}(\sigma)\right\|_{B\left(L_{\alpha}^{p}(\mathbb{K})\right)} \leq C_{1}^{t} C_{2}^{1-t}\|\sigma\|_{L_{\mu_{\alpha}}^{r}(\mathbb{R} \times \mathbb{K})},
$$

with

$$
\frac{t}{r}+\frac{1-t}{r^{\prime}}=\frac{1}{p}
$$


Theorem 4.10. Let $\sigma$ be in $L_{\mu_{\alpha}}^{r}(\mathbb{R} \times \mathbb{K}), r \in[1, \infty]$, and $h, k \in L_{\alpha}^{1}(\mathbb{K}) \cap L_{\alpha}^{\infty}(\mathbb{K})$. Then there exists a unique bounded linear operator

$$
\mathcal{L}_{h, k}(\sigma): L_{\alpha}^{p}(\mathbb{K}) \rightarrow L_{\alpha}^{p}(\mathbb{K})
$$

for all $p \in\left[\frac{2 r}{r+1}, \frac{2 r}{r-1}\right]$, and we have

$$
\left\|\mathcal{L}_{h, k}(\sigma)\right\|_{B\left(L_{\alpha}^{p}(\mathbb{K})\right)} \leq C_{3}^{\frac{t}{r}} C_{4}^{\frac{1-t}{r}}\left(C_{h} C_{k}\right)^{\frac{1}{2 r^{\prime}}}\|\sigma\|_{L_{\mu_{\alpha}}^{r}(\mathbb{R} \times \mathbb{K})},
$$

where

$$
\begin{aligned}
& C_{3}=\|k\|_{L_{\alpha}^{\infty}(\mathbb{K})}\|h\|_{L_{\alpha}^{1}(\mathbb{K})}, \\
& C_{4}=\|k\|_{L_{\alpha}^{1}(\mathbb{K})}\|h\|_{L_{\alpha}^{\infty}(\mathbb{K})},
\end{aligned}
$$

and

$$
t=\frac{r+1}{2}-\frac{r}{p}
$$

In order to prove this theorem we need the following lemmas.

Lemma 4.11. Let $\sigma$ be in $L_{\mu_{\alpha}}^{r}(\mathbb{R} \times \mathbb{K}), r \in[1, \infty], h \in L_{\alpha}^{2}(\mathbb{K}) \cap L_{\alpha}^{\infty}(\mathbb{K})$, and $k \in L_{\alpha}^{1}(\mathbb{K}) \cap L_{\alpha}^{2}(\mathbb{K})$. Then there exists a unique bounded linear operator

$$
\mathcal{L}_{h, k}(\sigma): L_{\alpha}^{\frac{2 r}{r+1}}(\mathbb{K}) \rightarrow L_{\alpha}^{\frac{2 r}{r+1}}(\mathbb{K})
$$

and we have

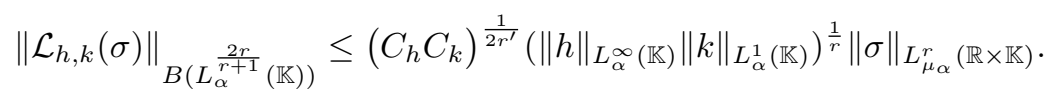

Proof. Consider the linear functional

$$
\begin{aligned}
\mathcal{I}: L_{\mu_{\alpha}}^{1}(\mathbb{R} \times \mathbb{K}) \cap L_{\mu_{\alpha}}^{\infty}(\mathbb{R} \times \mathbb{K}) & \rightarrow B\left(L_{\alpha}^{1}(\mathbb{K})\right) \cap B\left(L_{\alpha}^{2}(\mathbb{K})\right) \\
\sigma & \mapsto \mathcal{L}_{h, k}(\sigma) .
\end{aligned}
$$

Then by Proposition 4.1 and Theorem 3.5

$$
\|\mathcal{I}\|_{B\left(L_{\mu_{\alpha}}^{1}(\mathbb{R} \times \mathbb{K}), B\left(L_{\alpha}^{1}(\mathbb{K})\right)\right)} \leq\|h\|_{L_{\alpha}^{\infty}(\mathbb{K})}\|k\|_{L_{\alpha}^{1}(\mathbb{K})}
$$

and

$$
\|\mathcal{I}\|_{B\left(L_{\mu_{\alpha}}^{\infty}(\mathbb{R} \times \mathbb{K}), B\left(L_{\alpha}^{2}(\mathbb{K})\right)\right)} \leq \sqrt{C_{h} C_{k}}
$$

where $\|\cdot\|_{B\left(L_{\mu_{\alpha}}^{p}(\mathbb{R} \times \mathbb{K}), B\left(L_{\alpha}^{q}(\mathbb{K})\right)\right)}$ denotes the norm in the Banach space of the bounded linear operators from $L_{\mu_{\alpha}}^{p}(\mathbb{R} \times \mathbb{K})$ into $B\left(L_{\alpha}^{q}(\mathbb{K})\right), 1 \leq p, q \leq \infty$. Using an interpolation of 4.7) and 4.8 we get the result.

Lemma 4.12. Let $\sigma$ be in $L_{\mu_{\alpha}}^{r}(\mathbb{R} \times \mathbb{K}), r \in[1, \infty], k \in L_{\alpha}^{2}(\mathbb{K}) \cap L_{\alpha}^{\infty}(\mathbb{K})$, and $h \in L_{\alpha}^{1}(\mathbb{K}) \cap L_{\alpha}^{2}(\mathbb{K})$. Then there exists a unique bounded linear operator

$$
\mathcal{L}_{h, k}(\sigma): L_{\alpha}^{\frac{2 r}{r-1}}(\mathbb{K}) \rightarrow L_{\alpha}^{\frac{2 r}{r-1}}(\mathbb{K})
$$

and we have

$$
\left\|\mathcal{L}_{h, k}(\sigma)\right\|_{B\left(L_{\alpha}^{\frac{2 r}{r-1}}(\mathbb{K})\right)} \leq\left(C_{h} C_{k}\right)^{\frac{1}{2 r^{\prime}}}\left(\|h\|_{L_{\alpha}^{1}(\mathbb{K})}\|k\|_{L_{\alpha}^{\infty}(\mathbb{K})}\right)^{\frac{1}{r}}\|\sigma\|_{L_{\mu_{\alpha}}^{r}(\mathbb{R} \times \mathbb{K})} .
$$


Proof. As the adjoint of

$$
\mathcal{L}_{h, k}(\sigma): L_{\alpha}^{\frac{2 r}{r-1}}(\mathbb{K}) \rightarrow L_{\alpha}^{\frac{2 r}{r-1}}(\mathbb{K})
$$

is the bounded linear operator

$$
\mathcal{L}_{k, h}(\bar{\sigma}): L_{\alpha}^{\frac{2 r}{r+1}}(\mathbb{K}) \rightarrow L_{\alpha}^{\frac{2 r}{r+1}}(\mathbb{K}),
$$

the result follows from duality and the previous lemma.

Proof of Theorem 4.10. Using an interpolation of 4.6 and 4.9 , we have that, for any $p \in\left[\frac{2 r}{r+1}, \frac{2 r}{r-1}\right]$,

$$
\left\|\mathcal{L}_{h, k}(\sigma)\right\|_{B\left(L_{\alpha}^{p}(\mathbb{K})\right)} \leq C_{3}^{\frac{t}{r}} C_{4}^{\frac{1-t}{r}}\left(C_{h} C_{k}\right)^{\frac{1}{2 r^{\prime}}},
$$

with

$$
t=\frac{r+1}{2}-\frac{r}{p}
$$

Proposition 4.13. Let $p, r \in[1, \infty]$ be such that $p \in\left[\frac{2 r}{r+1}, 2\right]$. Let $\sigma \in L_{\mu_{\alpha}}^{r}(\mathbb{R} \times \mathbb{K})$, $h \in L_{\alpha}^{2}(\mathbb{K}) \cap L_{\alpha}^{\infty}(\mathbb{K})$, and $k \in L_{\alpha}^{1}(\mathbb{K}) \cap L_{\alpha}^{\infty}(\mathbb{K})$. Then there exists a unique bounded linear operator

$$
\mathcal{L}_{h, k}(\sigma): L_{\alpha}^{p}(\mathbb{K}) \rightarrow L_{\alpha}^{p}(\mathbb{K})
$$

and we have

$$
\left\|\mathcal{L}_{h, k}(\sigma)\right\|_{B\left(L_{\alpha}^{p}(\mathbb{K})\right)}\left(C_{h} C_{k}\right)^{\frac{1}{2 r^{\prime}}} C_{5}^{t} C_{6}^{1-t}\|\sigma\|_{L_{\mu_{\alpha}}^{r}(\mathbb{R} \times \mathbb{K})},
$$

where

$$
C_{5}=\left(\|h\|_{L_{\alpha}^{2}(\mathbb{K})}\|k\|_{L_{\alpha}^{2}(\mathbb{K})}\right)^{\frac{1}{q}}, \quad C_{6}=\|h\|_{L_{\alpha}^{\infty}(\mathbb{K})}\|k\|_{L_{\alpha}^{1}(\mathbb{K})},
$$

and

$$
t=\frac{(r-1) q}{(q-1) r}, \quad \text { with } \quad q=\frac{(2 p-2) r}{p-(2-p) r} .
$$

Proof. The proof follows from Theorem 4.8 and Theorem 3.5 with $p=1, q$ instead of $p$, and interpolation theory.

\subsection{Compactness of $\mathcal{L}_{h, k}(\sigma)$ for symbols in $L_{\alpha}^{p}(\mathbb{K})$.}

Proposition 4.14. Under the same hypothesis of Theorem 4.4, the localization operator

$$
\mathcal{L}_{h, k}(\sigma): L_{\alpha}^{1}(\mathbb{K}) \rightarrow L_{\alpha}^{1}(\mathbb{K})
$$

is compact.

Proof. Let $\left(f_{n}\right)_{n \in \mathbb{N}} \in L_{\alpha}^{1}(\mathbb{K})$ be such that $f_{n} \rightarrow 0$ weakly in $L_{\alpha}^{1}(\mathbb{K})$ as $n \rightarrow \infty$. It is enough to prove that

$$
\lim _{n \rightarrow \infty}\left\|\mathcal{L}_{h, k}(\sigma)\left(f_{n}\right)\right\|_{L_{\alpha}^{1}(\mathbb{K})}=0
$$


We have

$$
\begin{aligned}
& \left\|\mathcal{L}_{h, k}(\sigma)\left(f_{n}\right)\right\|_{L_{\alpha}^{1}(\mathbb{K})} \\
& \quad \leq \int_{\mathbb{K}} \int_{\mathbb{R} \times \mathbb{K}}|\sigma(a, x, t)|\left|\left\langle f_{n}, h_{a, x, t}\right\rangle_{L_{\alpha}^{2}(\mathbb{K})}\right|\left|k_{a, x, t}(s, y)\right| d \mu_{\alpha}(a, x, t) d \nu_{\alpha}(s, y) .
\end{aligned}
$$

Now using the fact that $f_{n} \rightarrow 0$ weakly in $L_{\alpha}^{1}(\mathbb{K})$, we deduce that

$$
\begin{aligned}
& \forall a \in \mathbb{R} \backslash\{0\}, \forall(x, t),(s, y) \in \mathbb{K}, \\
& \qquad \lim _{n \rightarrow \infty}|\sigma(a, x, t)|\left|\left\langle f_{n}, h_{a, x, t}\right\rangle_{L_{\alpha}^{2}(\mathbb{K})}\right|\left|k_{a, x, t}(s, y)\right|=0 .
\end{aligned}
$$

On the other hand, since $f_{n} \rightarrow 0$ weakly in $L_{\alpha}^{1}(\mathbb{K})$ as $n \rightarrow \infty$, there exists a positive constant $C$ such that $\left\|f_{n}\right\|_{L_{\alpha}^{1}(\mathbb{K})} \leq C$.

Hence by simple calculations we get, for all $a \in \mathbb{R} \backslash\{0\}$ and for all $(x, t),(s, y) \in \mathbb{K}$,

$$
|\sigma(a, x, t)|\left|\left\langle f_{n}, h_{a, x, t}\right\rangle_{L_{\alpha}^{2}(\mathbb{K})}\right|\left|k_{a, x, t}(s, y)\right| \leq C|\sigma(a, x, t)|\|h\|_{L_{\alpha}^{\infty}(\mathbb{K})}\left|\tau_{(x, t)}^{\alpha} k_{a}(s, y)\right| .
$$

Moreover, by Fubini's theorem and relation (2.7), we have

$$
\begin{aligned}
& \int_{\mathbb{K}} \int_{R \times \mathbb{K}}|\sigma(a, x, t)|\left|\left\langle f_{n}, h_{a, x, t}\right\rangle_{L_{\alpha}^{2}(\mathbb{K})}\right|\left|k_{a, x, t}(s, y)\right| d \mu_{\alpha}(a, x, t) d \nu_{\alpha}(s, y) \\
& \quad \leq C\|h\|_{L_{\alpha}^{\infty}(\mathbb{K})} \int_{\mathbb{R} \times \mathbb{K}}|\sigma(a, x, t)| \int_{\mathbb{K}}\left|\tau_{(x, t)}^{\alpha} k_{a}(s, y)\right| d \nu_{\alpha}(s, y) d \mu_{\alpha}(a, x, t) \\
& \quad \leq C\|h\|_{L_{\alpha}^{\infty}(\mathbb{K})} \int_{\mathbb{R} \times \mathbb{K}}|\sigma(a, x, t)| \int_{\mathbb{K}}\left|k_{a}(s, y)\right| d \nu_{\alpha}(s, y) d \mu_{\alpha}(a, x, t) \\
& \quad \leq C\|h\|_{L_{\alpha}^{\infty}(\mathbb{K})}\|k\|_{L_{\alpha}^{1}(\mathbb{K})}\|\sigma\|_{L_{\mu_{\alpha}}^{1}(\mathbb{R} \times \mathbb{K})}<\infty .
\end{aligned}
$$

Thus from the relations 4.10, 4.11, 4.12, 4.13, and the Lebesgue dominated convergence theorem we deduce that

$$
\lim _{n \rightarrow \infty}\left\|\mathcal{L}_{h, k}(\sigma)\left(f_{n}\right)\right\|_{L_{\alpha}^{1}(\mathbb{K})}=0
$$

and the proof is complete.

In the following we give two results for compactness of localization operators.

Theorem 4.15. Under the hypothesis of Theorem 4.4, the bounded linear operator

$$
\mathcal{L}_{h, k}(\sigma): L_{\alpha}^{p}(\mathbb{K}) \rightarrow L_{\alpha}^{p}(\mathbb{K})
$$

is compact for $1 \leq p \leq \infty$.

Proof. From the previous proposition, we only need to show that the conclusion holds for $p=\infty$. In fact, the operator

$$
\mathcal{L}_{h, k}(\sigma): L_{\alpha}^{\infty}(\mathbb{K}) \rightarrow L_{\alpha}^{\infty}(\mathbb{K})
$$

is the adjoint of the operator

$$
\mathcal{L}_{k, h}(\bar{\sigma}): L_{\alpha}^{1}(\mathbb{K}) \rightarrow L_{\alpha}^{1}(\mathbb{K})
$$


which is compact by the previous proposition. Thus by the duality property,

$$
\mathcal{L}_{h, k}(\sigma): L_{\alpha}^{\infty}(\mathbb{K}) \rightarrow L_{\alpha}^{\infty}(\mathbb{K})
$$

is compact. Finally, by an interpolation of the compactness on $L_{\alpha}^{1}(\mathbb{K})$ and on $L_{\alpha}^{\infty}(\mathbb{K})$, such as the one given on pages $202-203$ of the book [4] by Bennett and Sharpley, the proof is complete.

The following result is an analogue of Theorem 4.9 for compact operators.

Theorem 4.16. Under the hypotheses of Theorem 4.9, the bounded linear operator

$$
\mathcal{L}_{h, k}(\sigma): L_{\alpha}^{p}(\mathbb{K}) \rightarrow L_{\alpha}^{p}(\mathbb{K})
$$

is compact for all $p \in\left[r, r^{\prime}\right]$.

Proof. The result is an immediate consequence of an interpolation of Corollary 3.10 and Proposition 4.14. See again [4 pp. 202-203] for the interpolation used.

\section{ACKNOWLEDGEMENTS}

The authors are deeply indebted to the referees for providing constructive comments and help in improving the contents of this article. The first author thanks professor M. W. Wong for his help.

\section{REFERENCES}

[1] M. Assal and A. Rahmouni, An improved Hardy's inequality associated with the Laguerre Fourier transform, Collect. Math. 64 (2013), no. 2, 283-291. MR 3019365

[2] M. Assal and H. Ben Abdallah, Generalized Besov type spaces on the Laguerre hypergroup, Ann. Math. Blaise Pascal 12 (2005), no. 1, 117-145. MR 2126444.

[3] R. Atef, Uncertainty inequalities on Laguerre hypergroup, Mediterr. J. Math. 10 (2013), no. 1, 333-351. MR 3019109

[4] C. Bennett and R. Sharpley, Interpolation of operators, Pure and Applied Mathematics, 129, Academic Press, Boston, MA, 1988. MR 0928802

[5] A. P. Calderón, Intermediate spaces and interpolation, the complex method, Studia Math. 24 (1964), 113-190. MR 0167830

[6] C. K. Chui, An introduction to wavelets, Wavelet Analysis and its Applications, 1, Academic Press, Boston, MA, 1992. MR 1150048

[7] E. Cordero and K. Gröchenig, Time-frequency analysis of localization operators, J. Funct. Anal. 205 (2003), no. 1, 107-131. MR 2020210

[8] I. Daubechies, Ten lectures on wavelets, CBMS-NSF Regional Conference Series in Applied Mathematics, 61, Society for Industrial and Applied Mathematics (SIAM), Philadelphia, PA, 1992. MR 1162107

[9] I. Daubechies, Time-frequency localization operators: a geometric phase space approach, IEEE Trans. Inform. Theory 34 (1988), no. 4, 605-612. MR 0966733

[10] I. Daubechies and T. Paul, Time-frequency localisation operators - a geometric phase space approach. II. The use of dilations, Inverse Problems 4 (1988), no. 3, 661-680. MR 0965642

[11] I. Daubechies, The wavelet transform, time-frequency localization and signal analysis, IEEE Trans. Inform. Theory 36 (1990), no. 5, 961-1005. MR 1066587. 
[12] F. De Mari, H. G. Feichtinger and K. Nowak, Uniform eigenvalue estimates for time-frequency localization operators, J. London Math. Soc. (2) 65 (2002), no. 3, 720-732. MR 1895743

[13] F. De Mari and K. Nowak, Localization type Berezin-Toeplitz operators on bounded symmetric domains, J. Geom. Anal. 12 (2002), no. 1, 9-27. MR 1881289

[14] J. Faraut and K. Harzallah, Deux cours d'analyse harmonique: École d'été d'analyse harmonique de Tunis, 1984, Progress in Mathematics, 69, Birkhäuser Boston, Boston, MA, 1987. MR 898880

[15] G. B. Folland, Introduction to partial differential equations, second edition, Princeton University Press, Princeton, NJ, 1995. MR 1357411.

[16] P. Goupilland, A. Grossmann and J. Morlet, Cycle octave and related transforms in seismic signal analysis, Geoexploration 23 (1984), no. 1, 85-102. https://doi.org/10.1016/00167142(84)90025-5

[17] K. Gröchenig, Foundations of time-frequency analysis, Applied and Numerical Harmonic Analysis, Birkhäuser Boston, Boston, MA, 2001. MR 1843717.

[18] A. Grossmann and J. Morlet, Decomposition of Hardy functions into square integrable wavelets of constant shape, SIAM J. Math. Anal. 15 (1984), no. 4, 723-736. MR 0747432

[19] J. Huang, Sobolev space, Besov space and Triebel-Lizorkin space on the Laguerre hypergroup, J. Inequal. Appl. 2012, 2012:190, 19 pp. MR 3015422

[20] J. Huang, Littlewood-Paley $g$-functions and multipliers for the Laguerre hypergroup, J. Inequal. Appl. 2011, Art. ID 741095, 13 pp. MR 2772315

[21] T. H. Koornwinder, The continuous wavelet transform, in Wavelets: an elementary treatment of theory and applications, 27-48, Ser. Approx. Decompos., 1, World Sci. Publ., River Edge, NJ, 1993. MR 1238554

[22] L. Liu, A trace class operator inequality, J. Math. Anal. Appl. 328 (2007), no. 2, 1484-1486. MR 2290066

[23] G. Mauceri, Zonal multipliers on the Heisenberg group, Pacific J. Math. 95 (1981), no. 1, 143-159. MR 0631666

[24] H. Mejjaoli and K. Trimèche, Two-wavelet multipliers on the dual of the Laguerre hypergroup and applications, Mediterr. J. Math. 16 (2019), no. 5, Paper No. 126, 35 pp. MR 4000038.

[25] H. Mejjaoli and K. Trimèche, Localization operators associated with the Laguerre-Wigner transform, Ann. Univ. Ferrara Sez. VII Sci. Mat. 66 (2020), no. 2, 381-407. MR 4156201

[26] Y. Meyer, Wavelets and operators, Cambridge Studies in Advanced Mathematics, 37, Cambridge University Press, Cambridge, 1992. MR 1228209

[27] D. Müller, F. Ricci and E. M. Stein, Marcinkiewicz multipliers and multi-parameter structure on Heisenberg (-type) groups. I, Invent. Math. 119 (1995), no. 2, 199-233. MR 1312498

[28] S. Negzaoui, Lipschitz conditions in Laguerre hypergroup, Mediterr. J. Math. 14 (2017), no. 5, Paper No. 191, 12 pp. MR 3686827

[29] M. M. Nessibi and K. Trimèche, Inversion of the Radon transform on the Laguerre hypergroup by using generalized wavelets, J. Math. Anal. Appl. 208 (1997), no. 2, 337-363. MR 1441440

[30] J. Ramanathan and P. Topiwala, Time-frequency localization via the Weyl correspondence, SIAM J. Math. Anal. 24 (1993), no. 5, 1378-1393. MR 1234023

[31] E. M. Stein, Interpolation of linear operators, Trans. Amer. Math. Soc. 83 (1956), 482-492. MR 0082586

[32] K. Stempak, Mean summability methods for Laguerre series, Trans. Amer. Math. Soc. 322 (1990), no. 2, 671-690. MR 0974528 
[33] K. Stempak, An algebra associated with the generalized sublaplacian, Studia Math. 88 (1988), no. 3, 245-256. MR 0932012

[34] K. Trimèche, Generalized wavelets and hypergroups, Gordon and Breach Science Publishers, Amsterdam, 1997. MR 1489523.

[35] M. W. Wong, Wavelet transforms and localization operators, Operator Theory: Advances and Applications, 136, Birkhäuser Verlag, Basel, 2002. MR 1918652.

Hatem Mejjaoli ${ }^{凶}$

Taibah University, College of Sciences, Department of Mathematics, PO Box 30002, Al Madinah Al Munawarah, Saudi Arabia

hatem.mejjaoli@yahoo.fr

Khalifa Trimèche

University of Tunis El Manar, Faculty of Sciences of Tunis, Department of Mathematics, Campus, 2092 Tunis, Tunisia

khalifa.trimeche@gmail.com

Received: July 31, 2018

Accepted: September 28, 2019 